

University of Kentucky

UKnowledge

\title{
High-Excitation Emission Lines Near Eta Carinae, and Its Likely Companion Star
}

\author{
Andrea Mehner \\ University of Minnesota \\ Kris Davidson \\ University of Minnesota \\ Gary J. Ferland \\ University of Kentucky, gary@uky.edu \\ Roberta M. Humphreys \\ University of Minnesota
}

Follow this and additional works at: https://uknowledge.uky.edu/physastron_facpub

Part of the Astrophysics and Astronomy Commons, and the Physics Commons

Right click to open a feedback form in a new tab to let us know how this document benefits you.

\section{Repository Citation}

Mehner, Andrea; Davidson, Kris; Ferland, Gary J.; and Humphreys, Roberta M., "High-Excitation Emission Lines Near Eta Carinae, and Its Likely Companion Star" (2010). Physics and Astronomy Faculty Publications. 76.

https://uknowledge.uky.edu/physastron_facpub/76

This Article is brought to you for free and open access by the Physics and Astronomy at UKnowledge. It has been accepted for inclusion in Physics and Astronomy Faculty Publications by an authorized administrator of UKnowledge. For more information, please contact UKnowledge@lsv.uky.edu. 


\section{High-Excitation Emission Lines Near Eta Carinae, and Its Likely Companion Star Digital Object Identifier (DOI) \\ https://dx.doi.org/10.1088/0004-637X/710/1/729}

Notes/Citation Information

Published in The Astrophysical Journal, v. 710, no. 1, p. 729-742.

( 2 2010. The American Astronomical Society. All rights reserved. Printed in the U.S.A.

The copyright holder has granted permission for posting the article here. 


\title{
HIGH-EXCITATION EMISSION LINES NEAR ETA CARINAE, AND ITS LIKELY COMPANION STAR*
}

\author{
Andrea Mehner $^{1}$, Kris Davidson $^{1}$, Gary J. Ferland $^{2}$, and Roberta M. Humphreys ${ }^{1}$ \\ ${ }^{1}$ Department of Astronomy, University of Minnesota, Minneapolis, MN 55455, USA; mehner@astro.umn.edu, kd@astro.umn.edu, roberta@aps.umn.edu \\ ${ }^{2}$ Department of Physics and Astronomy, University of Kentucky, Lexington, KY 40506, USA; gjferland@googlemail.com \\ Received 2009 October 8; accepted 2009 December 15; published 2010 January 21
}

\begin{abstract}
In order to study the distribution of gas and ionizing radiation around $\eta$ Car and their implications for its likely companion star, we have examined high-excitation emission lines of [Ne III], [Fe III], etc., in spectra obtained with the Hubble Space Telescope/Space Telescope Imaging Spectrograph instrument during 1998-2004. Our principal results, some of them unexpected, are as follows. (1) The high-excitation fluxes varied systematically and nontrivially throughout $\eta$ Car's 5.5 year spectroscopic cycle. Instead of rising to a plateau after the 1998 "event," they changed continuously with a maximum in mid-cycle. (2) At one significant location a brief, strong secondary maximum occurred just before the 2003.5 spectroscopic event. (3) These emission lines are strongly concentrated at the "Weigelt knots" several hundred AU northwest of the star. With less certainty, [Ne III] appears to be somewhat more concentrated than [Fe III]. (4) A faster, blueshifted component of each feature appears concentrated near the star and elongated perpendicular to the system's bipolar axis. This structure may be related to the equatorial outflow and/or to dense material known to exist along our line of sight to the star. (5) Using the photoionization program Cloudy, we estimated the range of parameters for the hot secondary star that would give satisfactory high-excitation line ratios in the ejecta. $T_{\text {eff }} \approx 39,000 \mathrm{~K}$ and $L \sim 4 \times 10^{5} L_{\odot}$, for example, would be satisfactory. The allowed region in parameter space is wider (and mostly less luminous) than some previous authors suggested.
\end{abstract}

Key words: circumstellar matter - stars: emission line, $\mathrm{Be}$ - stars: individual (Eta Carinae) - stars: variables: general - stars: winds, outflows

\section{INTRODUCTION}

Eta Carinae is by far the most observable very massive star $\left(M>100 M_{\odot}\right)$, the only supernova impostor that can be observed in great detail, and it has other extraordinary attributes (see various papers in Humphreys \& Stanek 2005). The star and its ejecta produce more than 2000 identified emission lines, mostly of complex singly ionized species such as $\mathrm{Fe}^{+}$and $\mathrm{Ni}^{+}$ (Gaviola 1953; Aller \& Dunham 1966; Thackeray 1967; Viotti et al. 1989; Hamann et al. 1994; Zethson 2001). Satisfactory analyses of these low-ionization features have not been feasible, because they depend on radiative excitation processes which are extremely intricate. A few higher ionization spectral lines, however, offer valuable clues because they are less complicated: [Ne III], [Ar III], [Fe III], etc. They originate in slow-moving gas that was ejected from $\eta$ Car 100 to 200 years ago. For reasons explained below, they help to constrain the nature of a hot companion star which is thought to exist, but which has not been observed directly. They also give clues to the nature and distribution of ejecta within $3000 \mathrm{AU}$ of the central star. ${ }^{3}$

In this paper, we use Hubble Space Telescope (HST) data to explore the variability and spatial distribution of the highionization lines. Several notable results emerge, including maps of the emission, continuous evolution of the intensities through $\eta$ Car's 5.54 year spectroscopic cycle, and a suggestive blueshifted component of each line. Some of these facts contradict models that previously seemed credible. We also estimate limits to

\footnotetext{
* This research was supported by grant nos. GO-9973, 10844, and 11291 from the Space Telescope Science Institute. The HST is operated by the Association of Universities for Research in Astronomy, Inc., under NASA contract NAS5-26555.

3 Throughout this paper, if we omit individual references for either a minor detail or a well known generality about $\eta$ Car, see Humphreys \& Stanek

(2005), Gull et al. (2001), Morse et al. (1999), Davidson \& Humphreys (1997), and numerous references cited therein.
}

the secondary star's temperature and luminosity, based on the high-ionization features via photoionization calculations. We find a considerably broader range of allowed values than those proposed by Verner et al. (2005).

\subsection{Background Information and Astrophysical Motivations}

Several distinct types of spectra occur within $1^{\prime \prime}$ of $\eta$ Car. (1) The stellar wind produces broad emission and absorption lines, several hundred $\mathrm{km} \mathrm{s}^{-1}$ wide. (2) Slow-moving ejecta at $r \gtrsim 0$ '.15 (i.e., more than 300 AU from the star) emit features narrower than $50 \mathrm{~km} \mathrm{~s}^{-1}$. (3) The same ejecta contain dust which reflects the stellar-wind spectrum. (4) Faster ejecta also show both intrinsic and reflected spectra, though these are relatively faint in the central region. The high-excitation features belong mainly to category 2 , but their excitation mechanisms are quite different from the narrow low-ionization lines. The latter are strongly influenced by radiative processes such as resonance absorption of a UV photon leading to the emission of two or more lower energy photons. The high-ionization lines, however, depend on the same processes that dominate in ordinary nebulae-especially photoionization by a hot star. This star is most likely the companion object mentioned in Section 1.2.

The high-ionization lines have not been spatially mapped prior to this paper. Most of them are narrow and thus do not represent the $300-1000 \mathrm{~km} \mathrm{~s}^{-1}$ stellar wind. As a comparison, let us recall what is known about the spatial distribution of the lowexcitation features. The first $H S T$ spectra of $\eta$ Car showed that the narrow [Fe II] lines originate in the vicinity of the Weigelt knots, a set of three condensations $0{ }^{\prime \prime} 1-0{ }^{\prime} \cdot 3$ northwest of the star (Davidson et al. 1995, 1997; Weigelt \& Ebersberger 1986). These knots move outward rather slowly $\left(v \sim 40 \mathrm{~km} \mathrm{~s}^{-1}\right)$, probably near the equatorial plane of the system (Hofmann \& Weigelt 1988; Weigelt et al. 1995; Davidson et al. 1997; 
Zethson et al. 1999; Dorland et al. 2004; Smith et al. 2004). Later, Space Telescope Imaging Spectrograph (HST/STIS) data confirmed that low-excitation lines collectively account for much of the integrated brightness in that region (e.g., Gull et al. 1999; Johansson et al. 2000; Zethson et al. 1999). STIS could not resolve an individual Weigelt knot, but in cases where its slit crossed one of them, the spatial distribution of [Fe II] brightness peaked at the knot's position. Meanwhile many HST images showed the individual knots at various wavelengths, albeit not well resolved. Combining all these facts, most authors agree that the narrow low-excitation emission lines are largely concentrated in the Weigelt knots. The high-ionization features, on the other hand, had little influence on the HST images and on Weigelt's original speckle interferometry. ${ }^{4}$ Given their very different ionization states and excitation mechanisms, it is not safe to assume that they coincide with the knots. They might represent diffuse lower density gas surrounding the knots. This uncertainty inspired the main work reported in Section 3.

\subsection{Connections with $\eta$ Car's 5.54 Year Cycle, and the Secondary Star}

Observers noticed long ago that these high-excitation lines occasionally weaken or even disappear and then gradually return to their normal strength after a few months (Gaviola 1953; Thackeray 1967; Whitelock et al. 1983; Zanella et al. 1984). Zanella et al. interpreted such "spectroscopic events" as shell ejections that temporarily quash the ionizing UV flux. Eventually enough events had been observed to show that they recur with a period of 5.54 years (Damineli 1996; Whitelock et al. 1994; Damineli et al. 2008b). The 2-10 keV X-rays also disappear on each occasion (Ishibashi et al. 1999a, 1999b; Corcoran 2005). Many authors have discussed these phenomena, generally concluding that (1) there is a companion star in a highly eccentric 5.54 year orbit, (2) the X-rays are formed by the two colliding stellar winds, and (3) a spectroscopic event occurs near periastron due to a mass ejection and/or a disturbance in the wind and/or some sort of eclipse by the primary wind. The projected separation between stars is less than $30 \mathrm{AU} \sim 13$ mas, unresolvable by $H S T$, and the secondary star has not been directly detected (at least not in any unambiguous way). For details see many references in Humphreys \& Stanek (2005), Gull et al. (2001), and Morse et al. (1999), and the physical considerations listed by Martin et al. (2006b).

The simplest conventional theories do not predict much variation of the high-excitation lines between spectroscopic events. In those models the two stars are usually more than $10 \mathrm{AU}$ apart and the densest regions of the primary wind are usually on the far side of the secondary, from our point of view. Therefore, $\eta$ Car's spectrum at average times has received comparatively little attention. In Section 3, though, we find interesting behavior between the 1998.0 and 2003.5 events.

The secondary star's quantitative parameters remain open to question. The opaque wind of the primary star probably has a characteristic temperature below 20,000 K (Hillier et al. 2001)

\footnotetext{
4 Smith et al. (2000) asserted that HST/WFPC2 images with filters F631N and F658N showed [S III] and [N II] emission, respectively. However (1) the F658N images were overexposed in the region of interest here, and also included an indeterminate amount of redshifted $\mathrm{H} \alpha$ emission, and (2) there was no reason to assume that [S III] dominated in the F631N filter, which had been chosen to sample the continuum instead. STIS data show that in fact [S III] $\lambda 6314$ contributes less than $20 \%$ of the signal on the Weigelt knots. The WFPC2 F631N images show primarily the fluctuating continuum rather than [S III] emission.
}

too cool to account for the high-ionization lines. Its hypothetical companion, on the other hand, presumably must be a massive star in order to cause an event, but not as massive as the primary and therefore less evolved; so we expect it to be hot. The X-ray spectrum implies a $3000 \mathrm{~km} \mathrm{~s}^{-1}$ wind (Pittard \& Corcoran 2002), and such a fast wind almost always implies $T_{\text {eff }} \gtrsim 37,000 \mathrm{~K}$.

The usefulness of a hot companion for exciting $\eta$ Car's highionization lines has been recognized for more than a decade (Davidson 1997, 1999). This is an appealing idea because maybe we can deduce information about the secondary star from the high-excitation spectral features, via photoionization modeling. Since that object has not been observed directly, the $3000 \mathrm{~km} \mathrm{~s}^{-1}$ wind speed is almost our only other clue to its parameters. Verner et al. (2005) suggested spectral type and luminosity class $\mathrm{O} 7.5 \mathrm{I}$, but it is not clear that such an object would fulfill the requirements. We explore this question in Section 5.

\subsection{Contents of This Paper}

We restrict our analysis to a few well understood emission lines that require ionizing photons with $h v>16 \mathrm{eV}$. Using spectra obtained with the HST/STIS throughout an entire 5.54 year cycle, we examine the spatial distributions and temporal behavior of [Ne III] $\lambda 3870,[\mathrm{Fe}$ III] $\lambda \lambda 4659,4703$, and the narrow component of He I $\lambda 6680.5$ Then we calculate photoionization models to match the relative intensities of [Ne III], [Ar III], $\mathrm{He} \mathrm{I}$, and other lines that require helium-ionizing photons $(h v>25 \mathrm{eV})$.

In Section 2 we describe the $H S T /$ STIS data. The variability and distributions of the emission features are described in Section 3, while Section 4 concerns an interesting and significant blueshifted component seen in all of these lines. In Section 5 we describe our photoionization modeling with implications for the secondary star, and Section 6 concludes with a summary and brief discussion of our results.

\section{OBSERVATIONS, SPECTRAL ANALYSIS, AND MAPPING TECHNIQUE}

Eta Car was observed with HST/STIS from 1998.0 to 2004.2, covering an entire 5.54 year cycle. The first of these observations occurred on 1998 January 1, a few weeks after the most critical time in the 1998.0 spectroscopic event. The last of them were obtained on 2004 March 6, about eight months after the 2003.5 event. Most of the observations were concentrated in mid 2003.

This instrument provided the highest spatial resolution ever attained in spectroscopy of $\eta$ Car. The STIS/CCD had three large-format $(1024 \times 1024$ pixel $)$ detectors with 0 '.05 square pixels, covering a nominal $52^{\prime \prime} \times 52^{\prime \prime}$ square field of view and operating from below $2000 \AA$ to about $10000 \AA$. We used spectra observed with the $52^{\prime \prime} \times 00^{\prime} .1$ slit in combination with the G230MB, G430M, and G750M gratings which provided resolutions $R \sim 5000$ to 10,000 . The observations include a variety of slit positions and orientations, with a concentration at position angle $332^{\circ}$ where the star and Weigelt knots B and D all fell within the slit. ${ }^{6}$

\footnotetext{
5 Throughout this paper, we quote vacuum wavelengths and heliocentric Doppler velocities.

6 The star and the three main Weigelt knots are customarily labeled A, B, C, and D, with relative positions described by Weigelt \& Ebersberger (1986), Hofmann \& Weigelt (1988), Smith et al. (2004), and marked in our Figure 2. Their separations expand by about $1 \%$ per year.
} 
Table 1

Times of STIS Observations Used in the Emission Maps

\begin{tabular}{lccc}
\hline \hline \multicolumn{1}{c}{ Date (UT) } & MJD & Slit P.A. $^{\mathrm{a}}$ & $N_{\text {slit }^{\mathrm{b}}}$ \\
\hline 1998 Nov 25 $^{\mathrm{c}}$ & 51142 & $227^{\circ}$ & 1 \\
1999 Feb 22 & 51231 & $332^{\circ}$ & 1 \\
2000 Mar 21 & 51624 & $332^{\circ}$ & 1 \\
2001 Apr 18 & 52017 & $22^{\circ}$ & 1 \\
2001 Oct 1 & 52183 & $165^{\circ}$ & 1 \\
2002 Jan 20 & 52294 & $278^{\circ}$ & 1 \\
2002 Jul 5 & 52460 & $69^{\circ}$ & 2 \\
2003 Feb 13 & 52683 & $303^{\circ}$ & 1 \\
2003 Mar 29 & 52727 & $332^{\circ}$ & 1 \\
2003 May 5 & 52764 & $27^{\circ}$ & 1 \\
2003 May 17 & 52776 & $38^{\circ}$ & 2 \\
2003 Jun 2 & 52792 & $62^{\circ}$ & 2
\end{tabular}

Notes.

${ }^{\text {a }}$ P.A. $\pm 180^{\circ}$ gives same spatial coverage.

b The star was observed on every occasion, but sometimes there was also a second slit position offset about 0 '. $25 \mathrm{NW}$ of the star.

c There were no [Fe III] obs. on this date.

Altogether, a map of the slit locations resembles a quasirandom ensemble of intersecting lines (see the figures in Section 3 below).

$H S T / \mathrm{STIS} / \mathrm{CCD}$ data from the HST Treasury Project public archive $^{7}$ include several improvements over the normal Space Telescope Science Institute (STScI) pipeline and standard CALSTIS reductions. Modeling and interpolating the pixels in a special way improved the effective resolution along the crossdispersion axis, compared to most other published STIS/CCD data (Davidson 2006). In this process, the data were rebinned so that one pixel in the reduced data corresponds to 0.5 original CCD pixel, about 0.'025. Initial bad/hot pixel removal, wavelength calibration, and flux calibration matched or exceeded the STScI pipeline and CALSTIS. We extracted many local spectra centered at uniformly spaced locations along the STIS slit; the interval between successive locations was 0'.025 and each extraction was 0 .' 1 wide along the slit. (Characteristics of the original data precluded narrower extractions, see Davidson 2006.) At $\eta$ Car's distance $D \approx 2300$ pc (Davidson \& Humphreys 1997; Davidson et al. 2001; Smith 2006), 0.'1 corresponds to a projected size of about $230 \mathrm{AU}$.

We examined the spatial distribution and the temporal behavior of [ $\mathrm{Ne} \mathrm{III]} \lambda 3870,[\mathrm{Fe} \mathrm{III}] \lambda 4659$ and $\lambda 4703$, and the narrow component of $\mathrm{He}$ I $\lambda 6680$. These were selected for minimal contamination by adjacent emission or absorption features. ([Ne III] $\lambda 3870$, for instance, is weaker than [Ne III] $\lambda 3969$ but the latter nearly coincides with bright hydrogen and helium features.) For the spatial maps, we used data extending from 1998 November, 11 months after the 1998.0 event, to the beginning of 2003 June, a few weeks before the 2003.5 event (Table 1; we shall comment on the last dates below). We measured fluxes by integrating each line above the continuum, or in some cases above an underlying broad emission profile. Each measurement represented a spatial interval of about 0 .'1 along the slit, the extraction width mentioned above.

The assembly of a spatial map for each emission feature was a non-trivial exercise. Of course each STIS spectrogram sampled only the region covered by the slit, effectively a strip about 0 .'13 wide if we allow for HST's spatial resolution. On each observation date only one or two slit positions had been used, but

\footnotetext{
7 http://etacar.umn.edu/
}

these varied in orientation and some of them were offset from the star, so the whole ensemble sampled a useful fraction of the area within 0 '.5 of the star. Now consider any one of the measured emission lines. Subject to later verification, suppose that the feature's relative spatial distribution did not vary much-i.e., we tentatively assume that maps at any two different times closely resemble each other except for a normalization factor. (This statement does not apply to observations during an event, which we did not attempt to use.) Since the line's brightness varied through the spectroscopic cycle, we renormalized the values measured in each spectrogram so that the feature in question always had an adjusted value of unity at the only place that was observed on every occasion: the central star. Fortunately some narrow-line gas exists along our line of sight to the star, producing [Ne III], [Fe III], and $\mathrm{He}$ I lines superimposed on its spectrum. These reference measurements were less precise than those elsewhere, because the superimposed narrow emission features were weak compared to the underlying stellar spectrum. For each offset slit position, we adjusted the renormalization factor to maximize the consistency at intersections with other slit positions.

The above procedure obviously depends on some insecure assumptions, but we can apply consistency tests. Some of the slit positions passing through the star were close together or were used on more than one occasion, and each offset slit position intersected several of the others (see figures in Section 3). Consequently a substantial number of points were independently reobserved at well separated times. Note that each slit sample had only one adjustable parameter, its renormalization factor. Based on comparisons of the points re-observed in separate spectrograms, the overall self-consistency of each map turned out to be quite good, and we found no local inconsistency worse than the expected measurement errors. Therefore, our assumptions were sufficiently valid. ${ }^{8}$

Incidentally, possible time-delay effects cannot strongly influence these maps, since the light-travel time for 0.5 is only about a week at the distance of $\eta$ Car, and the cooling time in $\mathrm{a} \mathrm{He}^{+}$region is less than a month for electron densities $n_{e}>10^{5} \mathrm{~cm}^{-3}$.

In this paper, "phase" in the 5.54 year cycle is that used in the $\eta$ Car HST Treasury Program archive: $P=2023.0$ days, $t_{0}=$ MJD 50814.0 = J1998.0 exactly. This zero point corresponds to phase $\approx 0.009$ in a system proposed by Damineli et al. (2008b). Zero points based on specific phenomena in the spectroscopic events should be avoided for two reasons: the 1998.0-2003.52009.0 events differed in major respects, and, also, successive revisions of $t_{0}$ chosen in this way have made comparisons among papers more difficult. All proposed orbit models are far too imprecise to be useful in this regard. The Treasury Program phase definition, on the other hand, has been extant without alteration for a number of years and its period is consistent with observations. Considering that the Treasury Program archive is the largest available set of data on $\eta$ Car, it is the obvious reference standard.

\footnotetext{
8 These statements are true even for the data obtained a few weeks before the 2003.5 event. If more observations had been made in the mid-cycle period 1999-2002, we would have cautiously excluded the pre-event 2003 data, but fortunately the latter turn out to be consistent. On 2003 May 17 and June 2, the offset STIS slit intersected several earlier slit locations, see Table 1 and figures in Section 3. After the renormalization factor had been applied, line strengths matched reasonably well at those intersections.
} 


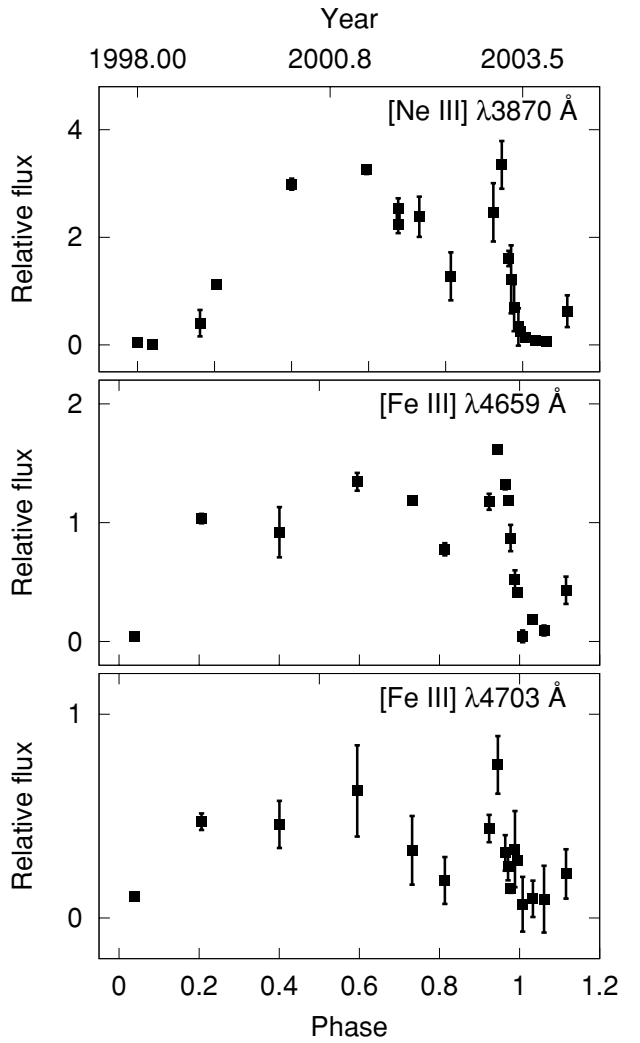

Figure 1. Strengths of the narrow [Ne III] $\lambda 3870$ and [Fe III] $\lambda \lambda 4659,4703$ emission lines measured in $H S T /$ STIS spectra of $\eta$ Car through its spectroscopic cycle. These represent gas along the line of sight to the star, seen with 0 .' 1 spatial resolution and not including the Weigelt knots. Features in the knots vary in a similar way.

\section{BEHAVIOR AND SPATIAL DISTRIBUTION OF THE EMISSION}

The different photon energies required for [Ne III], [Fe III], and $\mathrm{He}$ I emission allow us to probe a range of physical parameters. He I $\lambda 6680$ is a recombination line formed in a $\mathrm{He}^{+}$ region, while [Ne III] and [Fe III] arise in $\mathrm{Ne}^{++}$and $\mathrm{Fe}^{++}$zones. Ionization potentials of $\mathrm{H}^{0}, \mathrm{Fe}^{+}, \mathrm{He}^{0}, \mathrm{Ar}^{+}$, and $\mathrm{Ne}^{+}$are 13.6, 16.2, 24.6, 27.6, and $41.0 \mathrm{eV}$, respectively. Thus, for reasons explained by Osterbrock \& Ferland (2006), $\mathrm{Fe}^{++}$occurs mainly in regions of $\mathrm{H}^{+}$and $\mathrm{He}^{0}$, while $\mathrm{Ne}^{++}$and $\mathrm{Ar}^{++}$usually coexist with $\mathrm{He}^{+}$.

\section{1. [Ne III] Emission Line at $3870 \AA$}

The top panel of Figure 1 shows the time-dependent flux of the narrow [Ne III] $\lambda 3870$ emission line that was seen superimposed on STIS spectra of the star (at resolution $\sim 0^{\prime \prime} 1$ ) through the spectroscopic cycle 1998-2003. After a minimum lasting several months, the line intensity increased slowly until midcycle and then gradually decreased again. The intensity curve did not form a plateau. Then, a few months before the 2003.5 event, the line intensity rapidly increased again before falling off toward its minimum. This late-cycle rise resembled the behavior of the He II emission and the observed X-ray light curve (Ishibashi et al. 1999b; Martin et al. 2006b); perhaps the high-excitation emission at that time was somehow related to the X-rays or to the colliding-wind shocked zones (see Section 6 in Humphreys et al. (2008) for reasons why this might occur).

Some, but not all, of this behavior pattern was noted by Damineli et al. (2008a) for [Ar III] 27138 . They measured

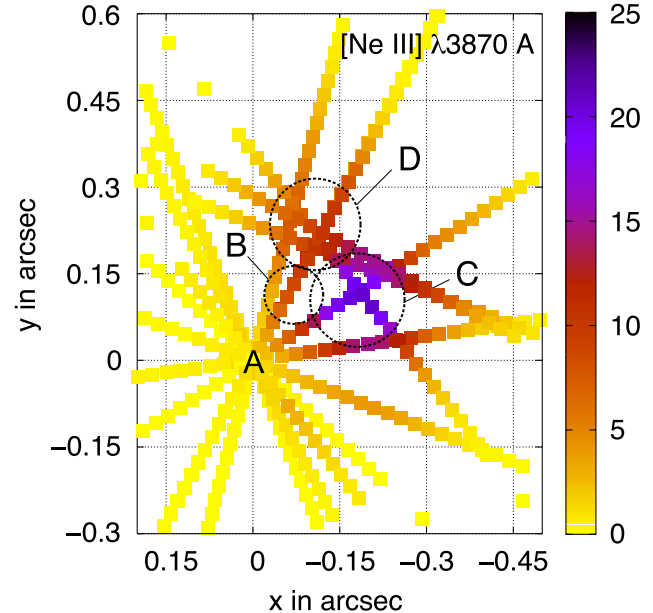

Figure 2. Spatial map of the narrow [Ne III] $\lambda 3870$ line. Position A marks the central star while B, C, and D are the Weigelt knots. Flux values are normalized so that this emission line's net flux is unity at the star's position.

equivalent widths (not fluxes) in ground-based spectra including the star plus ejecta out to $r \sim 1^{\prime \prime}$. Their Figure 1 differs from ours in two interesting respects. (1) The declines after mid-cycle do not match. At phase 0.8, for instance, the high-excitation lines had already fallen by $50 \%$ in the STIS results but less than $20 \%$ in the ground-based data. (2) Damineli et al. did not observe a brief, dramatic flux increase around phase 0.9. Most likely these discrepancies resulted from the very different spatial resolutions. The ground-based spectra include an amorphous unresolved mixture of emission regions, whereas our Figure 1 refers to a well defined 0 "'.1 locale which is particularly significant for a reason noted below.

A simple model would have predicted an extended plateau in Figure 1. The [Ne III] flux shown there is superimposed on the star and has a negative Doppler velocity of about $-40 \mathrm{~km} \mathrm{~s}^{-1}$; so it must represent slow-moving gas located approximately between us and the star. If it was ejected 50-200 years ago, this gas has now moved several hundred AU from the star. If the orbit orientation is within the range favored by most authors (Ishibashi 2001; Okazaki et al. 2008), then during most of the 5.5 year cycle the initial part of a path from the hot secondary star to the $\mathrm{Ne}^{++}$in question (i.e., toward us) should pass through the low-density secondary wind facing away from the primary star; see Figure 2 in Pittard \& Corcoran (2002). We omit quantitative details here, but previous data would have allowed a scenario in which the ionizing photons are not seriously depleted along such a path until they reach the observed $\mathrm{Ne}^{++}$region. In that case the [Ne III] brightness superimposed on the star would change little during most of the cycle. Figure 1 contradicts this simple model.

One can easily imagine qualitative explanations for the observed behavior after seeing it, but choosing the right one is harder. Most likely the ionizing photon path mentioned above does intersect a substantial and varying part of the dense primary wind. Gull et al. (2009) and Parkin et al. (2009), for instance, have discussed possible "conventional" models for the flow of dense gas outward from the binary system, allowing a substantial column density in the relevant sense. N. Soker (2009, private communication), on the other hand, remarks that Figure 1 is qualitatively consistent with a very different picture, in which the secondary star is usually on the far rather than the near side of the primary (Kashi \& Soker 2008, 2009b, 2009c). A discussion of these issues is beyond the scope of this paper, but 


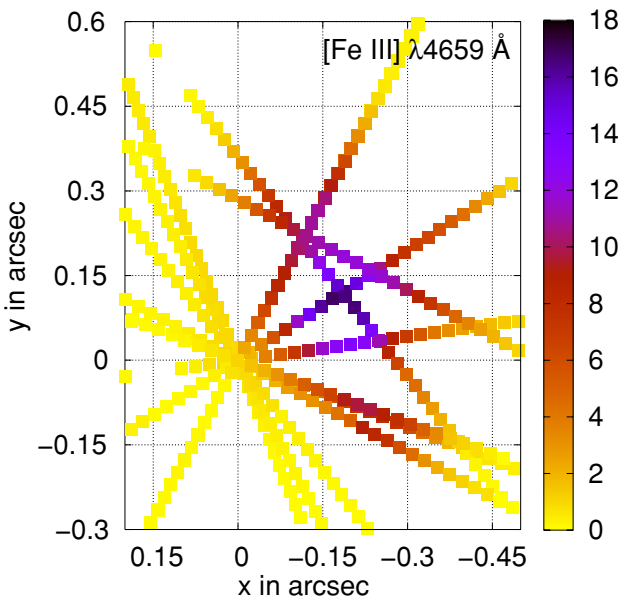

Figure 3. Spatial map of the narrow [Fe III] $\lambda 4659$ line, with the same format as Figure 2.

we note two points: (1) every proposed model depends on a number of assumptions which are not easy to verify, and (2) the information in Figure 1 is essential for any realistic view of the problem. Instead of an ill-defined average of ejecta around the star, this figure represents emission between us and the star at high spatial resolution.

Figure 2 shows a spatial map of the narrow [Ne III] $\lambda 3870$ feature in slit positions observed with STIS. In order to compensate for time variations, we renormalized the measurements so that the value is always unity at the position of the star (see Section 2). Most of the flux originates in the region of the Weigelt knots, at distances $0{ }^{\prime \prime} 1-00^{\prime} 3$ from the star. The positions of the rather amorphous knots BCD are marked in the figure. STIS could only marginally resolve them individually, but the peak obviously corresponds to Weigelt knot C. In our map, we can locate this peak at $r \approx 0.21$, position angle $\approx 300^{\circ}$. This corresponds well with measurements of knot C in HST images which gave $r \approx 0$ '.22 in 1999-2003 (e.g. Smith et al. (2004)).

Higher flux in [Ne III] $\lambda 3870$ was also observed at the locations of Weigelt knots $\mathrm{B}$ and $\mathrm{D}$ at position angle $\sim 335^{\circ}$. Their peak intensities are roughly half of that of knot $C$. However, it is not possible to distinguish between B and D based only on Figure 2.

Given the spatial-resolution limits of all HST data, the identification of [Ne III] with the Weigelt knots in Figure 2 is practically as good as the evidence for their association with [Fe II] reviewed in Section 1.1 above. Evidently the narrowline [Ne III] emission does not chiefly represent a diffuse halo enveloping all the Weigelt knots, as seemed possible before.

\section{2. [Fe III] Emission Lines at $4659 \AA$ and $4703 \AA$}

We examined both [Fe III] $\lambda 4659$ and $\lambda 4703$ in order to compare them for mutual consistency. Their theoretical intensity ratio is 1.85 (Nahar \& Pradhan 1996; Quinet 1996). Figures 3 and 4 show maps of the narrow [Fe III] $\lambda 4659$ and $\lambda 4703$ fluxes, respectively. In the same manner as for [Ne III], we rescaled the measured [Fe III] fluxes so the net value at the location of the star was always unity. Before rescaling, our measured flux of [Fe III] $\lambda 4659$ at that position was about 1.9 times as high as for [Fe III] $\lambda 4703$ in each observation, close to the theoretical value. The two [Fe III] maps are in excellent agreement with each other. [Fe III] emission originates in a slightly larger region than $[\mathrm{Ne}$ III]. This is not very surprising, given the difference

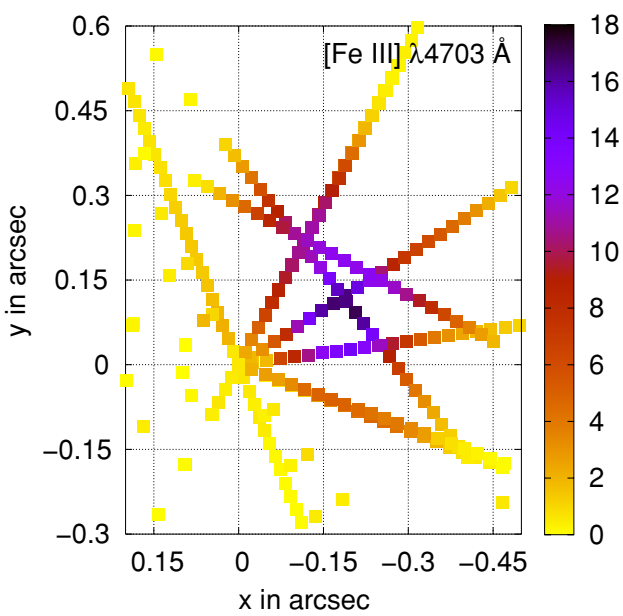

Figure 4. Similar to Figures $2-3$, but showing [Fe III] $\lambda 4703$. This map is based on the same spectrograms as Figure 3, but the measurements were independent.

in ionization potentials; [Ne III] occurs where helium is singly ionized but [Fe III] originates in zones where hydrogen is ionized but helium is not.

Incidentally, just as this paper was completed we noted a fainter additional $[\mathrm{Fe}$ III] condensation about 0 '.48 NNE of the star, in data obtained in 2009 June as part of a STIS recommissioning exercise. ${ }^{9}$ We mention this knot because it has a positive Doppler velocity, about $+27 \mathrm{~km} \mathrm{~s}^{-1}$ rather than the values around $-40 \mathrm{~km} \mathrm{~s}^{-1}$ seen in BCD. Its location was not sampled by the slit positions used in 1998-2004.

In addition to [Ne III], Figure 1 shows the temporal variation of [Fe III] $\lambda \lambda 4659,4703$ on the position of the star. They display the same qualitative behavior as [Ne III] $\lambda 3870$.

This paper is not directly concerned with low-excitation features, but we must acknowledge that some of the narrow $[\mathrm{Fe} \mathrm{II}]$ lines also showed temporary minima at phases around 0.8 in Figure 1, qualitatively like [Ne III] and [Fe III]. They all showed a peak at phase 0.9 and a brief minimum during the 2003 event. The [Fe II] lines varied differently from each other, but none of them showed a strong growth around phase 0.3 as [Ne III] did.

\subsection{He I Emission Line at $6680 \AA$}

He I $\lambda 6680$ is a recombination line formed in $\mathrm{He}^{+}$(Osterbrock $\&$ Ferland 2006). In a spectrum of $\eta$ Car, this feature usually consists of a broad component formed in the stellar wind, plus the narrow component from slow-moving ejecta. With the spectral resolution of STIS $\left(\sim 40 \mathrm{~km} \mathrm{~s}^{-1}\right)$, the underlying broad emission makes the narrow $\mathrm{He}$ I line far more difficult to measure than [Ne III] and [Fe III]. Consequently it is not a clear enough indicator of the time dependence to include in Figure 1. Our He I $\lambda 6680$ map (Figure 5) is thus of lower quality than the others. There is no evident disagreement compared to [Ne III].

\section{BLUESHIFTED COMPONENTS OF THE HIGH-EXCITATION LINES}

In addition to the narrow lines discussed above, [Fe III] and [Ne III] also show separate, broader components extending between Doppler velocities $-250 \mathrm{~km} \mathrm{~s}^{-1}$ and $-400 \mathrm{~km} \mathrm{~s}^{-1}$. These also exist for other high-excitation lines such as [Ar III],

\footnotetext{
9 HST program 11506: K. S. Noll, B. E. Woodgate, C. R. Proffitt, \& T. R. Gull.
} 


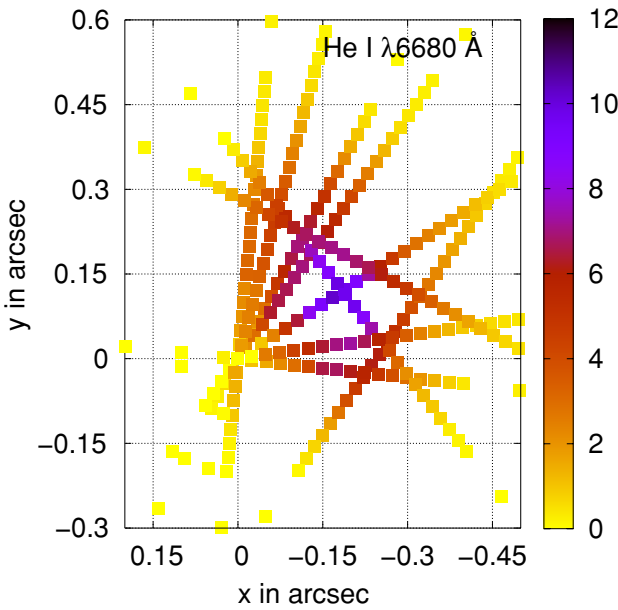

Figure 5. Map of the narrow He I $\lambda 6680$ emission line, shown in the same way as Figures 2-4.

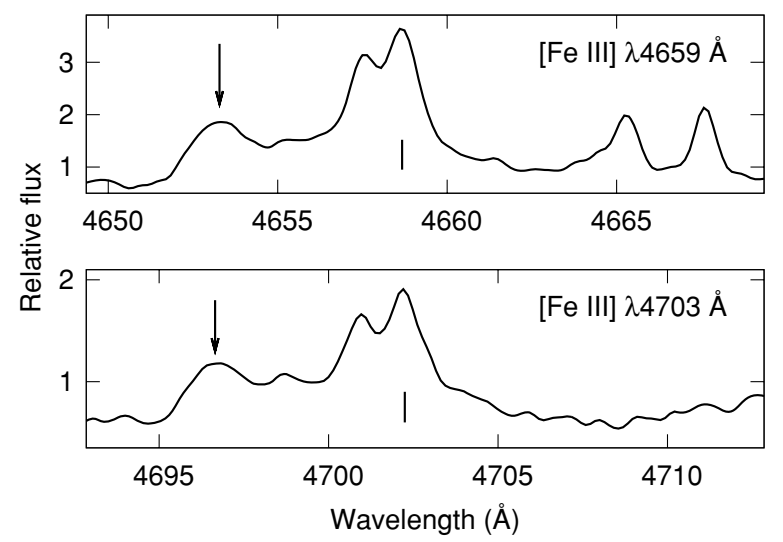

Figure 6. Profiles of the two [Fe III] emission lines that we measured at the position of the star. Arrows mark the broad negative-velocity emission and vertical ticks show the heliocentric zero-shift wavelengths.

[S III], [Si III], etc. In each case the blueshifted component is much wider than the narrow lines but much narrower than the normal stellar wind emission features. $(\eta$ Car's brightest Balmer lines usually have FWHM $\approx 400$ to $500 \mathrm{~km} \mathrm{~s}^{-1}$; see, e.g., Davidson et al. 2005)

Thackeray (1953) (1967) recognized [Fe III] and [S II] components as emission blueshifted by about $-300 \mathrm{~km} \mathrm{~s}^{-1}$. Aller \& Dunham (1966) listed these and similar blueshifted Fe II and [Fe II] lines. Zanella et al. (1984) noted that some of them disappeared along with the ordinary narrow high-excitation lines during an event, particularly including [Ne III]. They concluded that the blueshifted emission arose at larger distances from the star, but the Weigelt knots and polar/equatorial morphology were not known at that time. Comparing the 1992 spectroscopic event to the 1995 mid-cycle state, Damineli et al. (1998) confirmed the Zanella et al. statements about variability. Gull et al. (2009) recently showed STIS spectrograms (not tracings) of some of these features, but did not produce spatial maps like those presented below.

Quantitative measurement of the blueshifted [Ne III] $\lambda 3870$ is difficult due to nearby Si II $\lambda 3864$ and $\mathrm{Cr}$ II $\lambda 3867$ (Zethson 2001). We therefore concentrated on the blueshifted component of [Fe III] $\lambda 4659$, centered at about $4653 \AA$. To verify that it is [Fe III] emission, we compared the profiles of [Fe III] $\lambda 4659$ and $\lambda 4703$. Figure 6 shows that the blueshifted feature is undoubtedly a component of each [Fe III] line. This figure

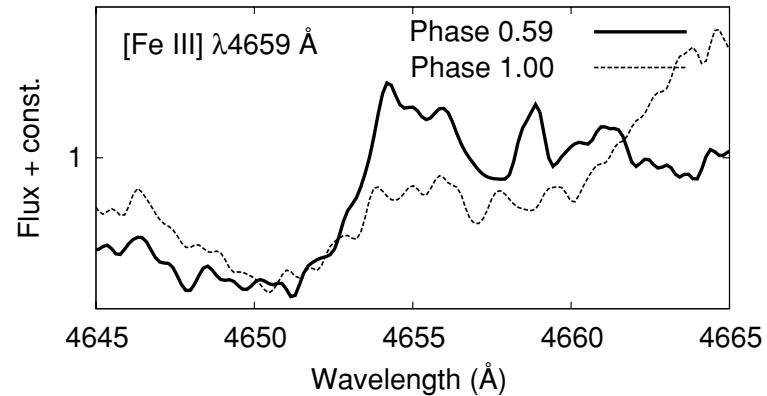

Figure 7. Comparison of the $[\mathrm{Fe}$ III] $\lambda 4659$ emission components at two different phases in the 5.54 year cycle. The solid line represents a phase of 0.59 near highexcitation maximum, while the dashed line shows phase 1.00 during the 2003.5 event.



Figure 8. Spatial map of the relative flux in the blue-shifted component of [Fe III] $\lambda 4659$. The flux is normalized to unity at the position of the star. When measured carefully the bright region is elongated along the NE-SW direction, see Figure 9.

also illustrates the complexity of the line profiles, tracing various ejection features. There is probably some material with intermediate Doppler shifts.

The temporal evolution of the blueshifted component follows the spectroscopic cycle in roughly the same way as the narrow lines. The normal and the shifted components appear and disappear together. Figure 7 compares the [Fe III] $\lambda 4659$ emission-line profile in spectra of the star at different phases in the cycle. The solid curve refers to a phase of 0.59 , close to the time when the narrow high-excitation emission was strongest. The dashed curve is the profile shortly after the 2003.5 event, at phase $\approx 1.0$. The narrow component at $4659 \AA$ Aisappeared completely, and the broad blue component was diminished.

Similar to Section 3, in Figures 8 and 9 we map the flux of the blueshifted component of [Fe III] $\lambda 4659$. Most of it originates in the inner 0 '. 1 region, less than $250 \mathrm{AU}$ from the star. The distribution is not as sharp as a stellar point source would be. The emission region is detectably elongated to the northeast and southwest, perpendicular to the axis of the bipolar Homunculus ejecta nebula. ${ }^{10}$

\footnotetext{
${ }^{10}$ Gull et al. (2009) mentioned "diffuse arcs" WSW and ENE of the star, but they meant velocity versus position correlations in spectrograms, not spatial arcs. "WSW" and "ENE" referred to the position angle of the STIS slit when those observations were made. Although they used direction names such as NE and ENE, those authors did not describe the quantitative elongation shown in our Figure 9. Their discussion focused instead on velocity-position-time effects that one can see directly in the spectrograms.
} 


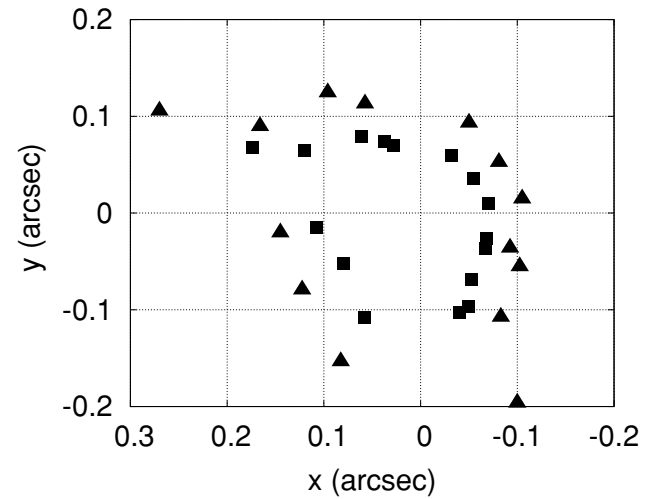

Figure 9. Expanded view of intensity contours of the blueshifted [Fe III] $\lambda 4659$, using the same measurements as Figure 8. Squares $(\square)$ and triangles $(\boldsymbol{\Delta})$ indicate values of 0.5 and 0.25 , respectively, relative to the value at the star's location. The contours are significantly elongated NE-SW, i.e., toward the upper left and lower right.

Considering the proximity of the blue component to the position of the star, its size, shape, and orientation, we suspect that Figure 9 traces the near side of a latitude-dependent structure in the outer stellar wind. Its observed blueshift of about $-300 \mathrm{~km} \mathrm{~s}^{-1}$ is smaller than the wind speed seen at the bottoms of the P Cyg absorption features (e.g., Smith et al. 2003; Davidson et al. 2005), but these two quantities sample the gas in very different ways. Electron densities cannot greatly exceed $10^{7} \mathrm{~cm}^{-3}$ in the relevant gas, because higher values would collisionally de-excite [Fe III], [Ne III], and [Ar III] too strongly (Section 5.3 below). Assuming that $\eta$ Car's mass-loss rate has the conventional value of $\sim 10^{-3} M_{\odot} \mathrm{yr}^{-1}$ and that its equatorial wind is less dense than its polar wind by a factor of order 3 (Smith et al. 2003), $n_{e}$ may fall below $10^{7} \mathrm{~cm}^{-3}$ at equatorial radii $r \gtrsim 100 \mathrm{AU} \sim 00^{\prime} 05$. At higher latitudes the corresponding radius would be larger. Since these size scales are much larger than the 5.54 year orbit, very likely the dense, biconical inner polar wind $(r<100 \mathrm{AU})$ shields the outer, lower density high-latitude zones from the secondary star's ionizing radiation. In other words, at high latitudes the ionizing radiation is probably absorbed by gas that is too dense to produce strong forbidden lines. Thus one expects the high-excitation forbidden lines to be concentrated toward latitudes less than, say, $50^{\circ}$ or so-resulting in an oblate emission region. If this picture is valid, evidently redshifted emission from the far side cannot penetrate through the configuration. Local inhomogeneities or "clumping" do not alter the basic reasoning. Gull et al. (2009) have recently discussed relevant time-dependent structures at smaller size scales. Their models depend on a number of assumptions and do not make use of a polar wind; but in general they have little effect on our comments above. Arguably the most significant well defined observational clue is the elongation shown in Figure 9-a new result.

On the other hand, we can imagine two other locations for emission farther from the star. First, the $-300 \mathrm{~km} \mathrm{~s}^{-1}$ Doppler shift is well matched to the region where our line of sight to the star intersects the inner parts of the bipolar Homunculus ejecta nebula and/or the "Little Homunculus" (Ishibashi et al. 2003). The main objection is that one might then expect the emission zone to have a projected size of 0.5 or larger. The other possibility involves a remarkable observed fact: our line of sight to the star has about two magnitudes more extinction than our view of the Weigelt knots (Davidson et al. 1995; Hamann et al. 1999; Hillier et al. 2001). Dust grains cannot exist much closer to $\eta$ Car than $r \sim 150 \mathrm{AU}$ (Davidson \& Humphreys 1997), but apparently some very localized dusty material lies between us and the star. Can its far side or inner side be the region where the blueshifted emission lines originate? Both the size scale in Figure 9 and the Doppler velocity seem reasonable. If this is the explanation, then the blueshifted line components must be intrinsically much brighter than the observable narrow lines but are obscured by the dust. (The same statement also applies to emission in the outer wind. This model and the outer wind idea mentioned earlier are not necessarily distinct from each other.)

\section{PHOTOIONIZATION MODELING AND THE NATURE OF THE SECONDARY STAR}

As mentioned in Section 1, the emission lines discussed here probably indicate photoionization by a hot star. Consider, for instance, [Ne III] $\lambda 3870$. This is a familiar feature in moderately high-excitation photoionized nebulae, where $\mathrm{Ne}^{++}$ typically exists in a $\mathrm{He}^{+}$zone maintained by ionizing photons with $25 \mathrm{eV}<h v<54 \mathrm{eV}$ (Osterbrock \& Ferland 2006). Within that zone, [Ne III] emission is collisionally excited by thermal electrons. Suppose we attempt to construct a non-photoionized model for the ejecta of $\eta$ Car. Since $\mathrm{Ne}^{+}$has an ionization potential of $41 \mathrm{eV}$, the creation of sufficient $\mathrm{Ne}^{++}$by thermal collisional ionization would require some mechanism-e.g., low-speed shock waves-that can heat a substantial amount of material to $T \sim 20,000-30,000 \mathrm{~K}$. This is difficult to achieve because a single low-speed shock carries insufficient energy, and no such mechanism has been suggested by other data. ${ }^{11}$

Moreover, as Zanella et al. (1984) emphasized, [Ne III] behaves very differently from the low-excitation lines during each spectroscopic event. Thus, as a very probable working hypothesis, we assume that [Ne III] $\lambda 3870$ signals ordinary, relatively straightforward quasi-nebular photoionization. So far as we know, no author has argued against this assumption in the case of $\eta$ Car. If correct then it is most likely true for [Ar III] and [Fe III] as well. The low-excitation features, by contrast, are strongly influenced by local absorption in various UV permitted lines-"radiative pumping"-which depends critically (and differently for each transition) on local velocity dispersions and density gradients. In other words, the high-excitation lines are theoretically more tractable than Fe II, [Fe II], and other lowexcitation features.

But where do the $\mathrm{Ne}^{+}$-ionizing photons above $41 \mathrm{eV}$ come from? Conventional models for the primary stellar wind are too cool (Hillier et al. 2001). The secondary star, however, is expected to have a high effective temperature. Its likely ability to create $\mathrm{He}^{+}$and $\mathrm{Ne}^{++}$in the ejecta was recognized as soon as its existence became probable (Davidson 1997, 1999). Since $\mathrm{Fe}^{++}, \mathrm{He}^{+}, \mathrm{Ar}^{++}$, and $\mathrm{Ne}^{++}$depend on stellar photon fluxes just above $16,25,27$, and $41 \mathrm{eV}$, the relative strengths of high-excitation lines may indicate the energy distribution of the star's ionizing UV photons, and thereby its temperature. Since the glare of the primary star makes this object impossible to study directly, practically the only other quantitative information on it comes from the X-ray spectrum and from evolutionary constraints. Therefore, here we

\footnotetext{
11 Shock fronts with $v \sim 30 \mathrm{~km} \mathrm{~s}^{-1}$ can produce the required temperature but the gas then cools rapidly. With densities $n_{e} \sim 10^{5}$ to $10^{8} \mathrm{~cm}^{-3}$ relevant in this paper, insufficient material would be hot unless it is shocked repeatedly, with a time interval less than a few months if $n_{e} \sim 10^{5} \mathrm{~cm}^{-3}$ or a few hr if $n_{e} \sim 10^{8} \mathrm{~cm}^{-3}$. A similar argument applies to heating by MHD waves. Strong shocks faster than $300 \mathrm{~km} \mathrm{~s}^{-1}$ are known to exist near $\eta$ Car but they ionize neon far beyond $\mathrm{Ne}^{++}$. Other considerations and quantitative details are too lengthy to discuss here.
} 
Table 2

Chemical Compositions

\begin{tabular}{lcc}
\hline \hline Element & \multicolumn{2}{c}{$\log _{10} \mathrm{~N}($ element $) / \mathrm{N}(\mathrm{H})$} \\
\cline { 2 - 3 } & Comp. $\mathrm{A}^{\mathrm{a}}$ & $\mathrm{Comp}^{\mathrm{b}} \mathrm{B}^{\mathrm{b}}$ \\
\hline $\mathrm{He}$ & -0.70 & -0.70 \\
$\mathrm{C}$ & -5.00 & -4.70 \\
$\mathrm{~N}$ & -3.10 & -2.80 \\
$\mathrm{O}$ & -5.00 & -4.70 \\
$\mathrm{Ne}$ & -4.00 & -3.70 \\
$\mathrm{Si}$ & -4.46 & -4.16 \\
$\mathrm{~S}$ & -4.74 & -4.44 \\
$\mathrm{Ar}$ & -5.60 & -5.30 \\
$\mathrm{Fe}$ & -4.55 & -4.25 \\
\hline
\end{tabular}

Notes.

a Default solar composition as used in Cloudy, except

$\mathrm{He}, \mathrm{C}, \mathrm{N}, \mathrm{O}$.

${ }^{\mathrm{b}} \mathrm{CNO}$ fraction somewhat larger than solar.

employ quasi-nebular photoionization calculations to model the relative high-excitation line strengths. Differences from earlier calculations described by Verner et al. (2005) will be noted in Section 5.4 .

\subsection{Procedure and Assumptions}

We used version 08.00 of the photoionization program Cloudy (Ferland et al. 1998) to simulate conditions observed at Weigelt knot C. Since the primary star can safely be neglected in the high-ionization zones, we assumed a simple configuration with one stellar source and a uniform-density cloud at distance $r \approx 10^{16} \mathrm{~cm} \approx 700 \mathrm{AU}$ which is reasonable for knot $\mathrm{C}$. We chose a covering factor of 0.05 and a filling factor of unity for the gas. We neglected possible UV extinction by dust within the $\mathrm{He}^{+}$region for three reasons: (1) local reddening of the [Fe II] emission is known to be small in the Weigelt knots (Hamann et al. 1999; Davidson et al. 1995) while the high-ionization zones should have smaller column densities; (2) dust grains associated with $\eta$ Car tend to be abnormally large (Davidson \& Humphreys 1997) and therefore relatively ineffective at $h v>20 \mathrm{eV}$, and (3) not enough information is available to realistically include the effects of local dust on the ionizing radiation. Altogether our assumptions represent an idealized view, but fortunately the line ratios used here depend only weakly on density and on geometrical details unless $n_{e}$ exceeds $10^{7} \mathrm{~cm}^{-3}$. A higher-density regime appears unlikely because collisional de-excitation would then suppress the forbidden lines too much. If $n_{e} \lesssim 10^{7} \mathrm{~cm}^{-3}$, the results depend mainly on the star's effective temperature $T_{\text {eff }}$ and the ionization parameter $U_{\mathrm{H}}$, see Section 5.3.

We assumed that the $\mathrm{He}^{+}$zone is ionization limited, i.e., that it absorbs nearly all incident photons above $25 \mathrm{eV}$. This assumption is based on three observed clues: (1) lower ionization regions clearly exist in and around the Weigelt knots, (2) almost no $\mathrm{He}$ I, [Ne III], and [Fe III] emission is seen at radii outside the locations of the knots, and (3) ratios of the He I, [Ne III], and [Fe III] lines do not vary much.

Studies of $\eta$ Car's outer ejecta show a peculiar chemical composition due to the $\mathrm{CNO}$ cycle. The gas is helium- and nitrogen-rich while carbon and oxygen are scarce (Davidson et al. 1986; Dufour et al. 1999). ${ }^{12}$

\footnotetext{
12 The stellar wind spectrum appears consistent with the outer ejecta but does
} not indicate abundances as well (Hillier et al. 2001).
Table 3

Selected High-excitation Emission Lines in Weigelt Blob C

\begin{tabular}{|c|c|c|c|c|c|}
\hline Spectrum & $\begin{array}{l}\lambda_{\text {vac }} \\
(\AA)\end{array}$ & $\begin{array}{c}\text { I.P.'s }{ }^{\mathrm{a}} \\
(\mathrm{eV})\end{array}$ & $\begin{array}{l}\text { EW } \\
(\AA) \\
\end{array}$ & $I_{\mathrm{obs}}{ }^{\mathrm{b}}$ & $I_{\text {corr }}{ }^{\mathrm{c}}$ \\
\hline [Ne III] & 3869.85 & $40.96-63.45$ & 10.24 & 3.63 & 1.00 \\
\hline [Si III] & 1892.03 & $16.34-33.49$ & 3.84 & 2.78 & 2.10 \\
\hline [Ar III] & 3110.08 & $27.63-40.74$ & 0.42 & 0.17 & 0.06 \\
\hline [Ar III] & 5193.26 & $27.63-40.74$ & 0.39 & 0.11 & 0.024 \\
\hline [Ar III] & 7137.76 & $27.63-40.74$ & 6.36 & 2.24 & 0.40 \\
\hline [Ar III] & 7753.24 & $27.63-40.74$ & 1.93 & 0.59 & 0.10 \\
\hline $\mathrm{He} \mathrm{I}$ & 4027.33 & $24.59-54.42^{\mathrm{d}}$ & 2.42 & 0.69 & 0.18 \\
\hline $\mathrm{He} \mathrm{I}$ & 6680.00 & $24.59-54.42^{\mathrm{d}}$ & 5.44 & 2.54 & 0.47 \\
\hline $\mathrm{He} \mathrm{I}$ & 7067.20 & $24.59-54.42^{\mathrm{d}}$ & 16.19 & 10.1 & 1.80 \\
\hline [S III $]$ & 6313.81 & $23.33-34.83$ & 7.72 & 2.39 & 0.45 \\
\hline [S III $]$ & 9071.11 & $23.33-34.83$ & 4.06 & 1.46 & 0.23 \\
\hline [S III $]$ & 9533.23 & $23.33-34.83$ & 5.49 & 2.49 & 0.39 \\
\hline [Fe III $]$ & 4659.35 & $16.18-30.65$ & 4.27 & 1.97 & 0.46 \\
\hline [Fe III $]$ & 4702.85 & $16.18-30.65$ & 3.21 & 0.88 & 0.21 \\
\hline
\end{tabular}

Notes.

Atomic data from http://physics.nist.gov/ PhysRefData/ASD/.

${ }^{a}$ Relevant ionization potentials. For instance, $\mathrm{Ne}^{+}$and $\mathrm{Ne}^{++}$have ionization potentials 40.96 and $63.45 \mathrm{eV}$.

b In units of $10^{-12} \mathrm{erg} \mathrm{cm}^{-2} \mathrm{~s}^{-1}$ within the $0^{\prime \prime} 1$ sampled area.

${ }^{c}$ De-reddened intrinsic strength relative to [Ne III] $\lambda 3870$.

${ }^{\mathrm{d}}$ Recombination spectrum created in $\mathrm{He}^{+}$zone.

We considered two sets of abundances, "compositions A and B" which differ by a factor of 2 for elements heavier than helium (Table 2). The mass fraction of $\mathrm{C}+\mathrm{N}+\mathrm{O}$ (mostly $\mathrm{N}$ ) is about $0.63 \%$ in composition $\mathrm{A}$ and $1.3 \%$ in $\mathrm{B}$. The former value resembles some crude observational estimates for $\eta$ Car (Davidson et al. 1986), while the latter is somewhat larger than Solar or Galactic material. The distinction between A and B turns out to affect the results only weakly, as explained in Section 5.3. Note that carbon and oxygen are too scarce to play an appreciable role.

We chose Weigelt knot $\mathrm{C}$ for this analysis because the highexcitation emission is strongest and best defined there, see Figure 2. Knot C was observed well with HST/STIS on 2003 February 13 (phase $=0.92$ in the 5.54 year cycle) and May 17 (phase = 0.97). We used data from 2003 February 13, obtained with STIS gratings G230MB, G430M, and G750M and with the slit passing through the star and knot $\mathrm{C}$ along position angle $303^{\circ}$. On that date the high-excitation lines were still strong, whereas by May 17 they had declined seriously as the 2003.5 event approached. We measured well isolated narrow high-excitation lines as target values for the photoionization models. The resulting equivalent widths and apparent intensities are listed in columns 4 and 5 of Table 3 . An error of $\pm 30 \%$ in an individual line strength, or perhaps even worse, would have little effect on our conclusions - partly because the photoionization models are very sensitive to the star's $T_{\text {eff }}$, and partly because the $\mathrm{He} / \mathrm{Ne} / \mathrm{Si} / \mathrm{Ar}$ abundance ratios are more uncertain than the observed line ratios.

We estimated the reddening from the [Ar III] $\lambda 3110 / \lambda 5193$ and [Ar III] $\lambda 7138 / \lambda 7753$ flux ratios. The intrinsic value of the former ratio, for instance, is reliably known because the $\lambda 3110$ and $\lambda 5193$ lines share the same upper level; and the case of $\lambda 7138 / \lambda 7753$ is similar. Other reddening indicators are less trustworthy because they use more than one ion species or differing upper levels or other model-dependent factors. Since no physical model is available for $\eta$ Car's anomalously large grains or for instrumental effects (see below), we adopted a 
standard approximation for wavelength-dependent extinction: $A_{\lambda} \approx a+b / \lambda$. For small amounts of reddening this choice of mathematical form is not critical, provided that coefficient $b$ is adjusted to give the right average slope from violet to red wavelengths. Fitting it to the [Ar III] measurements, we found surprisingly little reddening, $E_{B-V}=A_{B}-A_{V} \approx 0.2 \mathrm{mag}$.

This is only half the amount that the [Fe II] lines seem to indicate (Davidson et al. 1995; Hamann et al. 1999), but instrumental effects related to high spatial resolution probably account for most of the discrepancy. The normal wavelength dependence of HST's spatial resolution caused the slit throughput to decrease toward longer wavelengths for a localized source, and other effects also occurred in STIS data (Davidson 2006). Since these complications had smooth wavelength dependences, they are implicitly included in the effective or apparent reddening deduced from the [Ar III] line ratios. In other words, the $E_{B-V}$ value mentioned above was really the true interstellar and circumstellar value minus a correction for instrumental effects. Meanwhile it is also possible that the $[\mathrm{Fe} I \mathrm{II}$ method gives an overestimate of $E_{B-V}$. No matter which effect dominates, the corrected relative line strengths are automatically valid to sufficient accuracy because the [Ar III] comparison method is based on known intrinsic ratios. (As mentioned earlier, "sufficient accuracy" in this context would be $\pm 30 \%$ or allowably even worse. Most of the values in Table 3 are expected to be better than this.)

Thus, based on the [Ar III] lines, we adopted the following correction for net reddening:

$$
I_{\text {corr }}=0.105 \exp \left(\frac{3740 \AA}{\lambda}\right) I_{\text {obs }} .
$$

Here the constant factor, which has no effect on the line ratios used in our photoionization analysis, is adjusted to give [Ne III] $\lambda 3870$ a corrected value of 1.00 . As a result the corrected line strengths $I_{\text {corr }}$ are intrinsic values relative to this line. They are listed in the last column of Table 3.

\subsection{Stellar Model Atmospheres}

We explored a multidimensional grid of photoionization models, varying the effective stellar temperature, ionization parameter, and gas density. Our goal was to constrain these properties by comparing the calculated intensity ratios of highexcitation emission lines to the observed ones. We tried four theoretical stellar atmosphere grids available in Cloudy. The Atlas models are LTE, plane-parallel, hydrostatic atmospheres with turbulent velocity distribution $2 \mathrm{~km} \mathrm{~s}^{-1}$ (Castelli \& Kurucz 2004). The CoStar O-type models are non-LTE, line-blanketed model atmospheres, including stellar winds (Schaerer \& de Koter 1997). The Tlusty models are non-LTE, line-blanketed, plane-parallel, hydrostatic O-star spectral energy distribution (SEDs; Lanz \& Hubeny 2003). The WM-basic O-star grids represent non-LTE, line-blanketed, wind-blanketed hot stars (Pauldrach et al. 2001).

Unfortunately these four types of theoretical models disagree with each other in their UV spectral energy distributions. Figure 10 shows the continuum of an O-type main sequence star with $T_{\text {eff }}=40,000 \mathrm{~K}$ and $L=10^{5} L_{\odot}$ according to each model. Their differences are worst at high photon energies, particularly above $40 \mathrm{eV}$. Table 4 lists the total luminosities and photon rates that can ionize $\mathrm{H}^{0}, \mathrm{He}^{0}$, and $\mathrm{Ne}^{+}$in a comparable set of the four model types. The CoStar models do not include line opacity and therefore overestimate the far-UV flux because photon line absorption and subsequent re-emission at longer

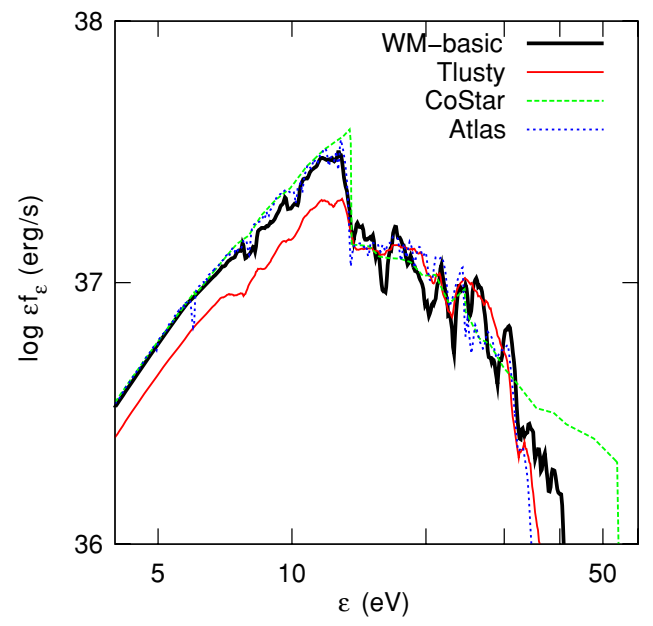

Figure 10. Comparison of the stellar continuum in Atlas, CoStar, Tlusty and WM-basic atmosphere models with $T_{\text {eff }}=40,000 \mathrm{~K}$. Tlusty and WM-basic models were smoothed.

wavelengths is not taken into account. The lower flux in the $\mathrm{He}^{0}$ continuum $(h v>25 \mathrm{eV})$ of the WM-basic model compared to the Atlas models is probably due to non-LTE effects producing deeper line cores in the blocking lines. The WM-basic code uses a consistent treatment of line blocking and blanketing (Smith et al. 2002). After experimenting with all four types, we adopted this type of model.

\subsection{Resulting Constraints on the Secondary Star}

The Weigelt knots encompass a wide range of densities (Hamann et al. 1999). We therefore varied the hydrogen density from $10^{5}$ to $10^{7} \mathrm{~cm}^{-3}$, and for each density we varied the ionization parameter $U_{\mathrm{H}}$ between $10^{-2}$ and $10^{2}$. In Cloudy, $U_{\mathrm{H}}$ is defined as the dimensionless quantity $Q_{\mathrm{H}} / 4 \pi r_{0}^{2} n_{\mathrm{H}} c$, where $Q_{\mathrm{H}}$ is the rate of hydrogen-ionizing photons $(h v>13.6 \mathrm{eV})$ emitted by the source star, $r_{0}$ is the distance from that source to the illuminated face of the cloud, $n_{\mathrm{H}}$ is the hydrogen density, and $c$ is the speed of light. ${ }^{13}$ For our purpose, of course, we care most about the photon supply above $25 \mathrm{eV}$ rather than $13.6 \mathrm{eV}$. Cloudy produces ionization-limited plane-parallel models, obviously a crude approximation to the true geometry.

Based on these calculations, Table 5 and Figure 11 show the effective temperatures of WM-basic stellar atmospheres that give the observed strengths of [Si III] $\lambda 1892$, [Ar III] $\lambda 7138$, and $\mathrm{He}$ I $\lambda 6680$ relative to $[\mathrm{Ne}$ III] $\lambda 3870$. ([Fe III] and [S III] are satisfactory in the models favored below.) For ionization parameters $\log U_{\mathrm{H}} \gtrsim+1$ the required stellar temperature is roughly constant, while smaller values of $\log U_{\mathrm{H}}$ require progressively higher $T_{\text {eff }}$. Since these results involve a subtle blend of uncertainties, the shaded regions in Figure 11 represent factor of 4 ranges for each line ratio. The best match to the observed data set occurs with $\log U_{\mathrm{H}}<-1$ and $T_{\text {eff }} \sim 40,000 \mathrm{~K}$. Here are some relevant considerations.

1. It is not surprising that chemical compositions A and B (Section 5.1) give similar results. Roughly speaking, the hydrogen and helium recombination lines indicate numbers of ionizing photons that have been absorbed, while the heavier-element emission lines account for much of the

\footnotetext{
13 The ionization parameter determines the sharpness of each ionization front, the coexistence of differing ionization stages, the ionized column density, and other physical attributes, as explained by Davidson \& Netzer (1979).
} 
Table 4

Comparison Between Different Stellar Atmosphere Models ${ }^{\mathrm{a}}$

\begin{tabular}{lccccc}
\hline \hline Model & $\begin{array}{c}L \\
\left(\mathrm{erg} / \mathrm{s}^{-1}\right)\end{array}$ & $\log L / L_{\odot}$ & $\begin{array}{c}\log Q_{\mathrm{H}} \\
\left(\text { photons s}^{-1}\right)\end{array}$ & $\begin{array}{c}\log Q_{\mathrm{He}} \\
\left(\text { photons s}^{-1}\right)\end{array}$ & $\begin{array}{c}\log Q_{\mathrm{Ne} \text { III }} \\
\left(\text { photons s}^{-1}\right)\end{array}$ \\
\hline Atlas & $3.67 \mathrm{e}+38$ & 4.98 & 48.72 & 48.27 & 46.09 \\
CoStar & $4.25 \mathrm{e}+38$ & 5.04 & 48.90 & 48.70 & 48.32 \\
Tlusty & $3.27 \mathrm{e}+38$ & 4.93 & 48.76 & 48.40 & 46.33 \\
WM-basic & $3.99 \mathrm{e}+38$ & 5.02 & 48.82 & 48.51 & 47.04 \\
\hline
\end{tabular}

Note. ${ }^{\mathrm{a}} T_{\text {eff }}=40,000 \mathrm{~K}, n_{\mathrm{H}}=10^{6} \mathrm{~cm}^{-3}$, and $\log U_{\mathrm{H}}=-1$

Table 5

WM-basic Model Predictions of $T_{\text {eff }}$ for Secondary Star and Composition A

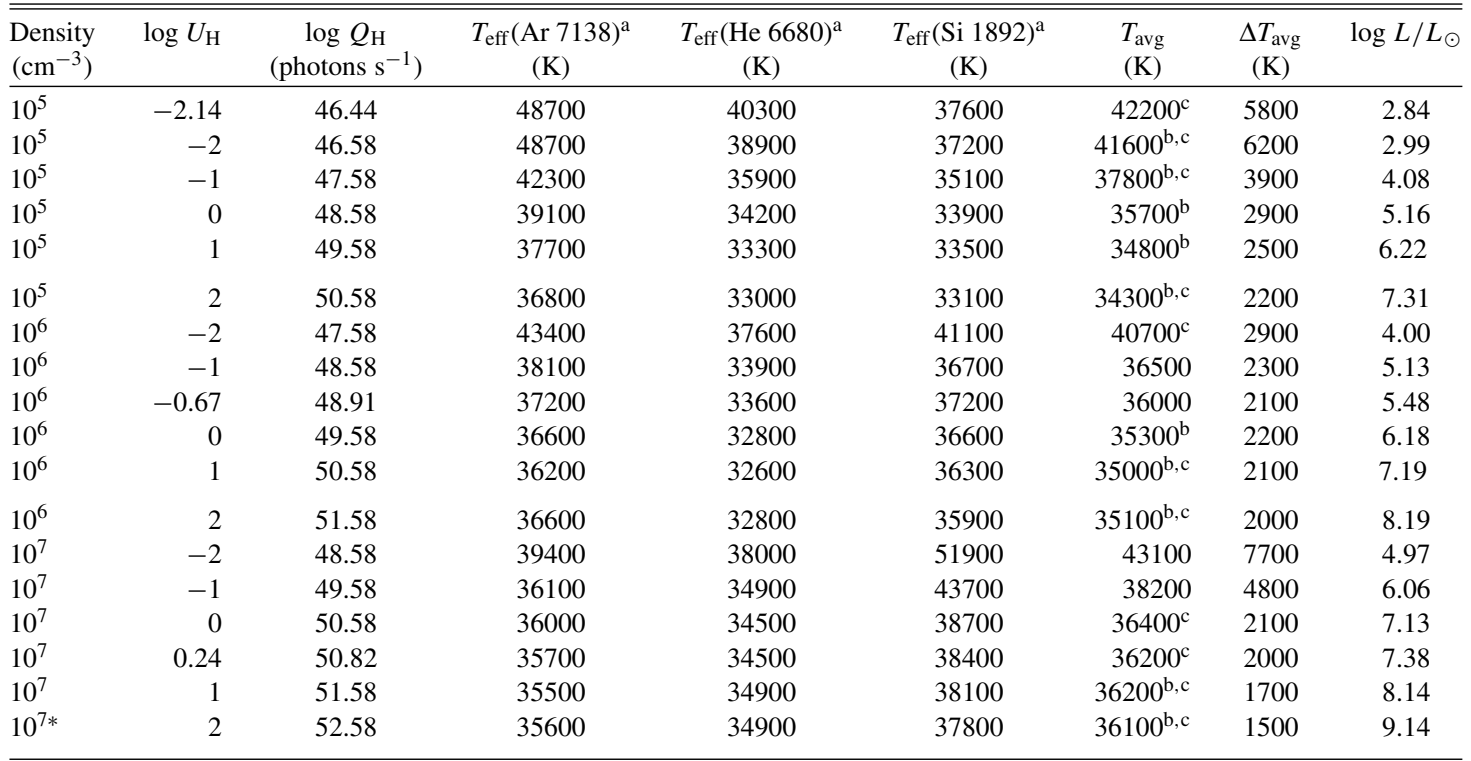

Notes.

a Relative to [Ne III] $\lambda 3870 \AA$.

${ }^{\mathrm{b}}$ Models with He II region larger than $4 \times 10^{15} \mathrm{~cm}$.

${ }^{\mathrm{c}}$ Models with $\log L / L_{\odot}$ smaller than 5 or larger than 6 .

cooling and thus represent the total energy in the absorbed photons (Davidson \& Netzer 1979; Osterbrock \& Ferland 2006). Therefore, if we alter the overall abundance of heavy elements relative to $\mathrm{H}+\mathrm{He}$, the equilibrium gas temperature automatically adjusts so that the ratio of heavy-element emission lines to hydrogen and helium lines does not change much. This ratio depends chiefly on the slope of the ionizing source's spectral energy distribution.

2. For $\log U_{\mathrm{H}}>-1$, He I $\lambda 6680$ indicates generally lower values of $T_{\text {eff }}$ than the other lines do. Very likely this clue is evidence for $\log U_{\mathrm{H}}<-1$; but we also note that models which are density limited, or limited by internal dust, or convex, can produce stronger helium lines relative to [Ne III].

3. Since relative abundances of individual heavy elements are quite uncertain in $\eta \mathrm{Car}$, and the reddening correction is uncertain for the UV [Si III] line, the systematic difference between [ArIII] and [SiIII] in Figure 11 is not very alarming.

4. Although the high-ionization emission lines are easier to model than low-ionization features, some special processes may occur. For example [Si III] $\lambda 1892$ may be enhanced by resonance-favored two-photon ionization of $\mathrm{Si}^{+}$(Johansson et al. 2006). Such effects are unusual, however, and unlikely to alter our basic conclusions. (If the [Si III] effect is strong, it produces a discrepancy between $\mathrm{He}$ I and [Si III] in photoionization models.)

5. Models with $n_{e} \gtrsim 2 \times 10^{7} \mathrm{~cm}^{-3}$ produce insufficient [Ne III] and [Ar III] emission compared to He I and [Si III], regardless of the stellar temperature. This is because the characteristic electron densities for collisional de-excitation of [Ne III] and [Ar III] are roughly $10^{7}$ and $5 \times 10^{6} \mathrm{~cm}^{-3}$, respectively.

6. Models with $n_{e} \ll 10^{6} \mathrm{~cm}^{-3}$ are ruled out by geometrical considerations. For reasons mentioned in Section 5.1, the $\mathrm{He}^{+}$region cannot be much smaller than the ionizationlimited thickness. This characteristic linear size $x$ is proportional to $U_{\mathrm{H}} / n_{e}$. In Table 5 and Figure 11, we have indicated models that are unsuitable because $x>250 \mathrm{AU}$, i.e., an ionization-limited $\mathrm{He}^{+}$zone would be larger than the region of the Weigelt knots. This criterion excludes all our models with $n_{e}=10^{5} \mathrm{~cm}^{-3}$, and those which have $n_{e}=10^{6} \mathrm{~cm}^{-3}$ and $\log U_{\mathrm{H}}>-0.65$.

7. A truly realistic model would include a range or distribution of gas densities, but we do not have enough observables to do this. At present one can say only that the "representative density" in a simplified model is very likely about half the maximum density which exists in the real, non-uniform $\mathrm{He}^{+}$ gas, in an order-of-magnitude sense. (This statement can be wrong if the configuration is unexpectedly complex.) 


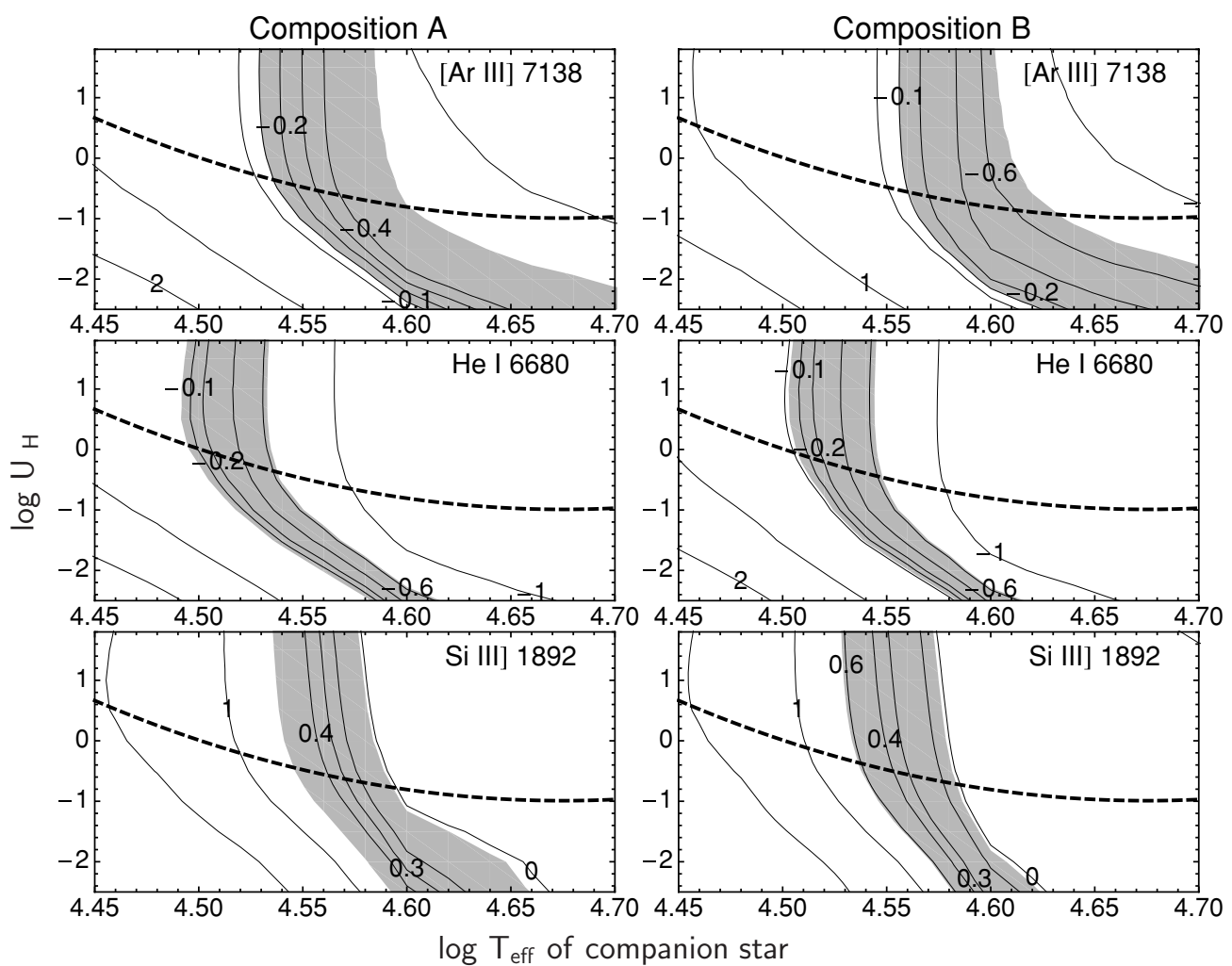

Figure 11. Contour plots of line intensity ratios [Ar III] $\lambda 7138 /[\mathrm{Ne}$ III] $\lambda 3870$ (top), He I $\lambda 6680 /[\mathrm{Ne}$ III] $\lambda 3870$ (middle), and Si III] $\lambda 1892 /[\mathrm{Ne}$ III] $\lambda 3870$ (bottom) in our photoionization models with $n_{\mathrm{H}}=10^{6} \mathrm{~cm}^{-3}$. Shaded areas correspond to the range of line intensity ratio between 0.5 and 2 times the observed value. Left and right columns refer to chemical compositions A and B, respectively. Models above the dashed curve are spatially too extended, violating criterion 6 in the text.

8. For a given gas density $n_{e}$ and stellar temperature $T_{\text {eff }}$, one can estimate the luminosity $L$ that would produce the assumed value of $U_{\mathrm{H}}$ in Weigelt knot C. As shown in the last column of Table 5, some choices of $\left(n_{e}, U_{\mathrm{H}}\right)$ lead to absurdly small or large values of $L$. Most important, the secondary star cannot exceed about $10^{6} L_{\odot}$ since its presence is not evident in the UV spectra obtained with $H S T /$ STIS. (The primary star has $L \approx 4 \times 10^{6} L_{\odot}$.)

The H-R diagram in Figure 12 summarizes our conclusions about the secondary star, assisted by a few more clues. Here two broad curves mark the results of our photoionization calculations with $n_{e}=10^{7} \mathrm{~cm}^{-3}$ and $10^{6} \mathrm{~cm}^{-3}$; the upper end of the latter curve is limited by criterion 6 above. If we assume that the two stars have the same age, then evolutionary circumstances further constrain the parameters. The minimum age for the primary star to have become helium-rich is $0.5 \mathrm{Myr}$ (Iben 1999), but it is most likely 2-3 Myr old based on its instabilities and probable association with the cluster Trumpler 16 (Walborn 1995). The lifetime of any star above $\sim 60 M_{\odot}$ is roughly $3 \mathrm{Myr}$. Therefore, in Figure 12 we show isochrones for $0.5,2$, and $3 \mathrm{Myr}$ as well as some evolutionary tracks, all adapted from Martins et al. (2005). We can base yet another constraint on the observed $\mathrm{X}$-ray spectrum, which indicates a secondary wind speed close to $3000 \mathrm{~km} \mathrm{~s}^{-1}$ (Pittard \& Corcoran 2002). Practically all stars with winds that fast have $T_{\text {eff }}>37,000 \mathrm{~K}$, marked by a vertical dotted line in Figure 12 (Kudritzki \& Puls 2000, and references cited therein).

Altogether, then, we expect the secondary star to lie somewhere within the shaded polygon in Figure 12, with the following limits. (1) The right-hand boundary is the X-ray-implied minimum temperature. Even without this argument we would deduce a similar limit from criterion 6 listed above for our pho-

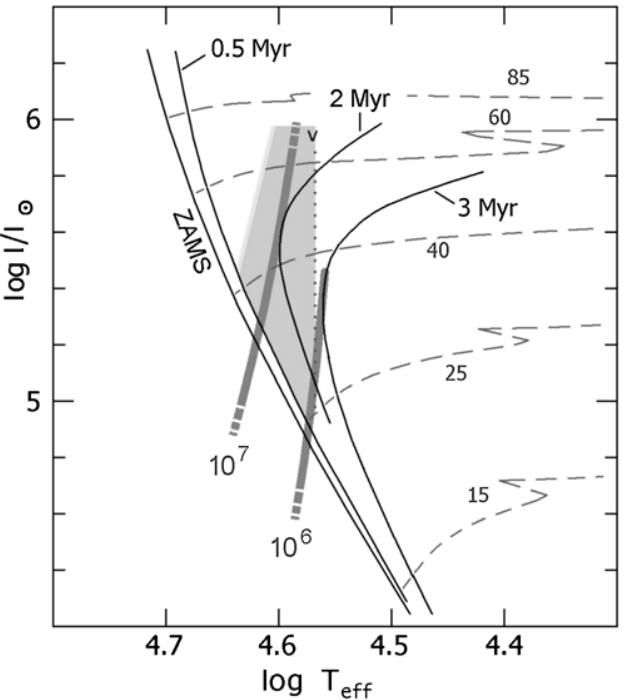

Figure 12. Likely positions for $\eta$ Car's secondary star in the H-R diagram. Solid curves show isochrones for $0.5,2$, and $3 \mathrm{Myr}$, while dashed curves show evolution tracks for initial masses 15 to $85 M_{\odot}$, all adapted from Martins et al. (2005). The two broad lines show $L$ vs. $T_{\text {eff }}$ correlations in our photoionization models with $n_{\mathrm{H}}=10^{7}$ and $10^{6} \mathrm{~cm}^{-3}$. A small "V" indicates a model suggested by Verner et al. (2005). The shaded polygon is the region allowed by various considerations (see the text).

toionization models. (2) The upper limit is $L \approx 10^{6} L_{\odot}$, criterion 8. (3) The upper-left boundary is based on our photoionization criterion $5, n_{e} \lesssim 2 \times 10^{7} \mathrm{~cm}^{-3}$. (4) The lower-left boundary is the $0.5 \mathrm{Myr}$ isochrone, and perhaps this limit should be moved rightward in the diagram to some age greater than $1 \mathrm{Myr}$. A star with $M_{\text {ZAMS }} \sim 40$ to $60 M_{\odot}$ and $T_{\text {eff }} \approx 40,000 \mathrm{~K}$, for example, would satisfy all these requirements. The corresponding spectral 
type would be $\mathrm{O} 4$ or O5 (Martins et al. 2005). If, however, the system is more than 2 Myr old, then the secondary's zero-age mass was probably less than $50 M_{\odot}$. (All statements related to isochrones obviously depend on the evolution models, though.)

If the entire region around $\eta$ Car were clearly visible, then the absolute brightnesses of the high-excitation emission lines would indirectly indicate the secondary star's luminosity. In fact, the local extinction is far too patchy, but we attempted a crude estimate based on the observable [Ne III] brightness. In our Cloudy calculations with reasonable input parameters, the ionizing star's luminosity was around 2300 times the luminosity of $[\mathrm{Ne} \mathrm{III}] \lambda 3870$. Measuring the total flux of this line in the Weigelt knots, and assuming reasonable factors for the extinction and the solid angle intercepted by the knots, we found $\log L / L_{\odot} \sim 5.5$. This estimate is very rough, but it is consistent with Figure 12 and thereby suggests that the absolute fluxes are reasonable.

Pittard \& Corcoran (2002) assumed that the secondary star has a mass-loss rate close to $10^{-5} M_{\odot} \mathrm{yr}^{-1}$, in order to obtain sufficient X-ray luminosity in their colliding-wind models. Such a high rate would be very unusual, and not entirely consistent with our discussion above. Possibly this is an argument in favor of a more luminous secondary star, but on the other hand the mass-loss estimate is not very robust. Only a small fraction of the wind's kinetic energy is converted to observable X-rays, via some complicated efficiency factors. The secondary wind speed, however, almost directly determines the average temperature seen in the $2-10 \mathrm{keV} \mathrm{X}$-ray spectrum. This is why we consider the $3000 \mathrm{~km} \mathrm{~s}^{-1}$ speed estimate to be relatively more trustworthy than the mass-loss rate.

One can imagine models that are seriously affected by changes in the secondary star. For instance, conceivably it was originally more massive but has now become a Wolf-Rayet object. Soker (2007) has proposed another scenario, wherein the secondary star accreted a large amount of mass during the great eruption 160-170 years ago, and has not yet returned to its normal thermal equilibrium. (See also Kashi \& Soker (2009a).) Apart from obvious complications and a lack of substantive evidence for them, models of these types are clearly not the simplest possibilities. They have multiple adjustable parameters, and thus are beyond the scope of this paper. (Note, incidentally, that our photoionization calculations do not favor an extremely hot star with $T_{\text {eff }}>45,000 \mathrm{~K}$.)

\subsection{Comparison with Previous Calculations}

Verner et al. (2005) reported an earlier set of photoionization calculations very much like ours, with the same goal, but they deduced appreciably different parameters for the secondary star. Like us, they used Cloudy with WM-basic atmospheres, uniform density in the ionized material, etc. They used earlier STIS data, but the high-excitation line ratios were similar to ours within the uncertainties. Verner et al. appear to have supposed that the high-ionization lines originate much closer to the star than the locations of the Weigelt knots, but this probably had little effect on their results. They described photoionization models with specific sets of parameters, but did not show systematic maps of parameter space as in our Table 5 and Figures 11 and 12.

There are three notable differences between their results and ours. The allowed region in Figure 12 is much larger than their discussion seems to imply, their suggested upper limit for $T_{\text {eff }}$ is barely above our lower limit, and they assigned a maximal luminosity to the secondary star. Indeed Verner et al. proposed that it has $T_{\text {eff }} \approx 37,200 \mathrm{~K}$ and $\log L / L_{\odot} \approx 5.97$, marked with a small "V" in our Figure 12. They classified it "O7.5 I," but according to Martins et al. (2005) an O5.5 supergiant would have that temperature. Such an object is not excluded by the calculations, but in our view it has three disadvantages. It has the minimum temperature required for a $3000 \mathrm{~km} \mathrm{~s}^{-1}$ wind, requires the age of the system to be less than $2 \mathrm{Myr}$, and has practically the largest allowable luminosity. So far as we know, there is no strong argument against lower luminosities. In summary, given the limited information currently available (Section 5.3 above), the parameters suggested by Verner et al. are not the most suitable choices for assessing the nature of $\eta$ Car's companion at this time.

\section{SUMMARY}

Here we have reported the behavior and the spatial origins of $\eta$ Car's quasi-nebular high-excitation emission between spectroscopic events. Neither of these questions was previously explored with adequate spatial and temporal sampling. Indeed the 1998-2004 HST/STIS spectra appear to constitute the only suitable existing data set. Thus, our main results were previously vague or unknown, and some of them contradict models that would otherwise have seemed credible.

For instance, after the 1998 spectroscopic event the highexcitation features did not simply recover to constant "normal" intensities, but instead varied systematically throughout the 5.5 year cycle (Sections 3.1, 3.2). [Ne III] and [Fe III] evolved through four distinct stages (Figure 1).

1. As expected based on previous events, the weak or near-zero state persisted for several months. This was long enough for a companion star to sweep through more than $180^{\circ}$ of longitude around periastron, if its orbit eccentricity is at least 0.8 as seems likely.

2. Subsequent growth to near-maximum intensity was interestingly slow, delaying the maximum until more than two years after the event-unlike what a simple model would predict.

3. Then, instead of leveling off, the intensity soon began to decline, following a sort of parabolic trajectory in time.

4. A few months before the 2003.5 event, however, it briskly rose to a second maximum just before declining again to near zero.

We directly measured the double-peaked cycle in gas along the line of sight to the star, but at least stages 1-3 seem broadly valid in our data on the Weigelt knots BCD as well. As noted in Section 3.1, Damineli et al. (2008a) recognized those stages for one [Ar III] line at much lower spatial resolution, but there is a quantitative disagreement in stage 3 and they did not see stage 4 .

If this time pattern was caused by some complex variation of the circumstellar extinction, then in order to include BCD it must extend out to $r \gtrsim 800 \mathrm{AU}$, which seems unlikely in terms of grain physics. Apparently, then, during most of the 1998-2003 cycle the amounts of $\mathrm{Ne}^{+2}, \mathrm{Fe}^{+2}$, etc. in BCD were significantly less than their maxima. Despite our comments at the beginning of Section 5, the secondary maximum might conceivably be related to the colliding-wind shock structure which was very strong in the months before an event. The softest X-rays may play a role then (Martin et al. 2006b). A detailed speculative/ theoretical interpretation of Figure 1 would require calculations beyond the scope of this paper. Martin et al. (2006a, 2009) have described other phenomena observed midway between $\eta$ Car's 
spectroscopic events, emphasizing that its mid-cycle behavior deserves more attention.

In Section 3, we also showed spatial maps of the [Ne III] and [Fe III] emission in the sub-arcsecond vicinity of $\eta$ Car. Informal examinations of [Ar III], [S III], and [Si III] gave similar impressions. Evidently high-excitation emission is strongly concentrated in the Weigelt knots BCD located at a distances 0.'15-0.'35 northwest of the star, rather than in a surrounding halo or in the region between the star and the knots as seemed possible before. Prior to this work most researchers would very likely have assumed that each knot produces high-excitation emission, but here we have given the first clear proof. Weigelt blob $\mathrm{C}$ has the highest flux. Maps of the various ion species all show basically the same picture, except that those with higher ionization potentials seem a little more compact. Unfortunately it will be difficult to improve these maps in the future, because the rising brightness of the central star is rapidly making the knots harder to observe (Martin et al. 2006c).

The high-excitation lines all have blueshifted components in the velocity range -250 to $-400 \mathrm{~km} \mathrm{~s}^{-1}$. This fact was known before, but in Section 4 we have shown that (1) it originates mostly within $0{ }^{\prime \prime} 15$ of the central star, and (2) this region is elongated along the equatorial (not polar) axis of the $\eta$ Car system. In Section 4, we proposed several plausible interpretations involving the three-dimensional distribution of stellar ejecta. These alternatives are not mutually exclusive.

Assuming that a hot companion star is responsible for the narrow high excitation emission lines, in Section 5 we found constraints on its parameters based on photoionization calculations. Our results are consistent with evolutionary considerations and, independently, with the colliding-wind X-ray temperature. Figure 12 shows that the allowed region in parameter space is larger than some previous authors suggested. For example, an O4-O6 giant with $L \sim 4 \times 10^{5} L_{\odot}, T_{\text {eff }} \approx 39,000 \mathrm{~K}$, and $M_{\text {ZAMS }} \sim 40-50 M_{\odot}$ would fall near the center of the allowed range. Our calculations did not exclude moderately higher masses and luminosities, but neither did they favor them.

This research made use of numerous $H S T$ observations and was partially supported by grants 10844 and 11291 from the Space Telescope Science Institute (STScI), which is operated by the Association of Universities for Research in Astronomy, Inc., under NASA contract NAS 5-26555. The data archive for the HST Treasury Program on Eta Carinae (HST program GO 9973) is available online at http://etacar.umn.edu/, partially supported by the University of Minnesota and STScI. Our analyses used software tools developed for the Treasury Program by K. Davidson, K. Ishibashi, and J. C. Martin. Calculations were performed with version 08.00 of the photoionization program Cloudy described by Ferland et al. (1998). Finally, we thank the referee, N. Soker, for pointing out some logical gaps and pertinent references that we neglected in the original version.

\section{REFERENCES}

Aller, L. H., \& Dunham, T. J. 1966, ApJ, 146, 126

Castelli, F., \& Kurucz, R. L. 2004, arXiv:astro-ph/0405087

Corcoran, M. F. 2005, AJ, 129, 2018

Damineli, A. 1996, ApJ, 460, L49

Damineli, A., Stahl, O., Kaufer, A., Wolf, B., Quast, G., \& Lopes, D. F. 1998, A\&AS, 133, 299

Damineli, A., et al. 2008a, MNRAS, 386, 2330

Damineli, A., et al. 2008b, MNRAS, 384, 1649

Davidson, K. 1997, New Astron., 2, 387
Davidson, K. 1999, in ASP Conf. Ser. 179, Eta Carinae at The Millennium, ed. J. A. Morse, R. M. Humphreys, \& A. Damineli (San Francisco, CA: ASP), 304

Davidson, K. 2006, in The 2005 HST Calibration Workshop: Hubble After the Transition to Two-Gyro Mode, ed. A. M. Koekemoer, P. Goudfrooij, \& L. L. Dressel (Baltimore, MD: STScI), 247

Davidson, K., Dufour, R. J., Walborn, N. R., \& Gull, T. R. 1986, ApJ, 305, 867

Davidson, K., Ebbets, D., Johansson, S., Morse, J. A., \& Hamann, F. W. 1997, AJ, 113, 335

Davidson, K., Ebbets, D., Weigelt, G., Humphreys, R. M., Hajian, A. R., Walborn, N. R., \& Rosa, M. 1995, AJ, 109, 1784

Davidson, K., \& Humphreys, R. M. 1997, ARA\&A, 35, 1

Davidson, K., \& Netzer, H. 1979, Rev. Mod. Phys., 51, 715

Davidson, K., Smith, N., Gull, T. R., Ishibashi, K., \& Hillier, D. J. 2001, AJ, 121, 1569

Davidson, K., et al. 2005, AJ, 129, 900

Dorland, B. N., Currie, D. G., \& Hajian, A. R. 2004, AJ, 127, 1052

Dufour, R. J., Glover, T. W., Hester, J. J., Currie, D. G., van Orsow, D., \& Walter, D. K. 1999, in ASP Conf. Ser. 179, Eta Carinae at The Millennium, ed. J. A. Morse, R. M. Humphreys, \& A. Damineli (San Francisco, CA: ASP), 134

Ferland, G. J., Korista, K. T., Verner, D. A., Ferguson, J. W., Kingdon, J. B., \& Verner, E. M. 1998, PASP, 110, 761

Gaviola, E. 1953, ApJ, 118, 234

Gull, T. R., Ishibashi, K., Davidson, K., \& The Cycle 7 STIS Go Team. 1999, in ASP Conf. Ser. 179, Eta Carinae at The Millennium, ed. J. A. Morse, R. M. Humphreys, \& A. Damineli (San Francisco, CA: ASP), 144

Gull, T. R., Johannson, S., \& Davidson, K. (ed.) 2001, ASP Conf. Ser. 242, Eta Carinae and Other Mysterious Stars: The Hidden Opportunities of Emission Spectroscopy (San Francisco, CA: ASP)

Gull, T. R., et al. 2009, MNRAS, 396, 1308

Hamann, F., Davidson, K., Ishibashi, K., \& Gull, T. R. 1999, in ASP Conf. Ser. 179, Eta Carinae at The Millennium, ed. J. A. Morse, R. M. Humphreys, \& A. Damineli (San Francisco, CA: ASP), 116

Hamann, F., Depoy, D. L., Johansson, S., \& Elias, J. 1994, ApJ, 422, 626

Hillier, D. J., Davidson, K., Ishibashi, K., \& Gull, T. 2001, ApJ, 553, 837

Hofmann, K.-H., \& Weigelt, G. 1988, A\&A, 203, L21

Humphreys, R., \& Stanek, K. (ed.) 2005, ASP Conf. Ser. 332, The Fate of the Most Massive Stars (San Francisco, CA: ASP)

Humphreys, R. M., Davidson, K., \& Koppelman, M. 2008, AJ, 135, 1249

Iben, I., Jr. 1999, in ASP Conf. Ser. 179, Eta Carinae at The Millennium, ed. J. A. Morse, R. M. Humphreys, \& A. Damineli (San Francisco, CA: ASP), 367

Ishibashi, K. 2001, in ASP Conf. Ser. 242, Eta Carinae and Other Mysterious Stars: The Hidden Opportunities of Emission Spectroscopy, ed. T. R. Gull, S. Johannson, \& K. Davidson (San Francisco, CA: ASP), 53

Ishibashi, K., Corcoran, M. F., Davidson, K., Swank, J. H., Petre, R., Drake, S. A., Damineli, A., \& White, S. 1999a, ApJ, 524, 983

Ishibashi, K., Davidson, M. F., Corcoran, K., Drake, S. A., Swank, J. H., \& Petre, R. 1999b, in ASP Conf. Ser. 179, Eta Carinae at The Millennium, ed. J. A. Morse, R. M. Humphreys, \& A. Damineli (San Francisco, CA: ASP), 266

Ishibashi, K., et al. 2003, AJ, 125, 3222

Johansson, S., Hartman, H., \& Letokhov, V. S. 2006, A\&A, 452, 253

Johansson, S., Zethson, T., Hartman, H., Ekberg, J. O., Ishibashi, K., Davidson, K., \& Gull, T. 2000, A\&A, 361, 977

Kashi, A., \& Soker, N. 2008, MNRAS, 390, 1751

Kashi, A., \& Soker, N. 2009a, New Astron., 14, 11

Kashi, A., \& Soker, N. 2009b, MNRAS, 394, 923

Kashi, A., \& Soker, N. 2009c, MNRAS, 397, 1426

Kudritzki, R.-P., \& Puls, J. 2000, ARA\&A, 38, 613

Lanz, T., \& Hubeny, I. 2003, ApJS, 146, 417

Martin, J. C., Davidson, K., Hamann, F., Stahl, O., \& Weis, K. 2006a, PASP, 118,697

Martin, J. C., Davidson, K., Humphreys, R. M., Hillier, D. J., \& Ishibashi, K. 2006b, ApJ, 640, 474

Martin, J. C., Davidson, K., Humphreys, R. M., \& Mehner, A. 2009, ApJ, submitted (arXiv:0908.1627)

Martin, J. C., Davidson, K., \& Koppelman, M. D. 2006c, AJ, 132, 2717

Martins, F., Schaerer, D., \& Hillier, D. J. 2005, A\&A, 436, 1049

Morse, J. A., Humphreys, R. M., \& Damineli, A. (ed.) 1999, ASP Conf. Ser. 179, Eta Carinae At The Millennium (San Francisco, CA: ASP)

Nahar, S. N., \& Pradhan, A. K. 1996, A\&AS, 119, 509

Okazaki, A. T., Owocki, S. P., Russell, C. M. P., \& Corcoran, M. F. 2008, MNRAS, 388, L39

Osterbrock, D. E., \& Ferland, G. J. (ed.) 2006, Astrophysics of Gaseous Nebulae and Active Galactic Nuclei (Sausalito, CA: Univ. Science Books) 
Parkin, E. R., Pittard, J. M., Corcoran, M. F., Hamaguchi, K., \& Stevens, I. R. 2009, MNRAS, 394, 1758

Pauldrach, A. W. A., Hoffmann, T. L., \& Lennon, M. 2001, A\&A, 375, 161

Pittard, J. M., \& Corcoran, M. F. 2002, A\&A, 383, 636

Quinet, P. 1996, A\&AS, 116, 573

Schaerer, D., \& de Koter, A. 1997, A\&A, 322, 598

Smith, L. J., Norris, R. P. F., \& Crowther, P. A. 2002, MNRAS, 337, 1309

Smith, N. 2006, ApJ, 644, 1151

Smith, N., Davidson, K., Gull, T. R., Ishibashi, K., \& Hillier, D. J. 2003, ApJ, 586,432

Smith, N., Morse, J. A., Davidson, K., \& Humphreys, R. M. 2000, AJ, 120, 920

Smith, N., et al. 2004, ApJ, 605, 405

Soker, N. 2007, ApJ, 661, 490

Thackeray, A. D. 1953, MNRAS, 113, 211
Thackeray, A. D. 1967, MNRAS, 135, 51

Verner, E., Bruhweiler, F., \& Gull, T. 2005, ApJ, 624, 973

Viotti, R., Rossi, L., Cassatella, A., Altamore, A., \& Baratta, G. B. 1989, ApJS, 71,983

Walborn, N. R. 1995, RevMexAA Conf. Ser., 2, 51

Weigelt, G., \& Ebersberger, J. 1986, A\&A, 163, L5

Weigelt, G., et al. 1995, RevMexAA Conf. Ser., 2, 11

Whitelock, P. A., Feast, M. W., Carter, B. S., Roberts, G., \& Glass, I. S. 1983, MNRAS, 203, 385

Whitelock, P. A., Feast, M. W., Koen, C., Roberts, G., \& Carter, B. S. 1994 MNRAS, 270, 364

Zanella, R., Wolf, B., \& Stahl, O. 1984, A\&A, 137, 79

Zethson, T. 2001, PhD thesis, Lunds Univ., Sweden

Zethson, T., Johansson, S., Davidson, K., Humphreys, R. M., Ishibashi, K., \& Ebbets, D. 1999, A\&A, 344, 21 